\title{
Berezin transform on the quantum unit ball
}

\author{
Dmitry Shklyarov ${ }^{\dagger} \quad$ Genkai Zhang ${ }^{\ddagger}$ \\ ${ }^{\dagger}$ Institute for Low Temperature Physics \& Engineering \\ 47 Lenin Ave., 61103 Kharkov, Ukraine \\ ${ }^{\ddagger}$ Chalmers Tekniska Högskola/Göteborgs Universitet, Matematik \\ 412 96, Göteborg, Sweden \\ e-mail: shklyarov@ilt.kharkov.ua, genkai@math.chalmers.se
}

\begin{abstract}
We introduce and study, in the framework of a theory of quantum Cartan domains, a $q$-analogue of the Berezin transform on the unit ball. We construct $q$-analogues of weighted Bergman spaces, Toeplitz operators and covariant symbol calculus. In studying the analytical properties of the Berezin transform we introduce also the $q$-analogue of the $S U(n, 1)$-invariant Laplace operator (the Laplace-Beltrami operator) and present related results on harmonic analysis on the quantum ball.. These are applied to obtain an analogue of one result by A. Unterberger and H. Upmeier. An explicit asymptotic formula expressing the $q$-Berezin transform via the $q$-Laplace-Beltrami operator is also derived. At the end of the paper, we give an application of our results to basic hypergeometric $q$-orthogonal polynomials.
\end{abstract}

\section{Introduction}

Since appearance of quantum groups in the middle of 1980's there have been different attempts to find an appropriate generalization to the $q$-case of various classical constructions in analysis and geometry of Lie groups. Recently, it became clear [1] that there should exist a substantial $q$-analogue of the theory of Cartan domains (the most studied class of such domains constitute the so-called classical domains [2]). In turn, this observation has opened a way to generalization of other important theories about those domains. One of the most fascinating among them is the Berezin's quantization [3]. Though Berezin applied his construction to a wide class of Kähler manifolds, the most complete and precise results have being obtained just in the particular case of Cartan domains [4, 5]. 
It should be noted that first attempts to find $q$-analogues of some constructions of the Berezin's theory were made before the study of $q$-Cartan domains has being initiated. For example, in [6] the authors studied a two-parameter deformation $\mathcal{P}_{q, \lambda}$ of the polynomial algebra $\mathbb{C}[z, \bar{z}]$ related to certain $S U(1,1)$-covariant Poisson brackets on the unit disk. They showed among other things that for any $\lambda$ the algebra $\mathcal{P}_{q, \lambda}$ is acted upon by the quantum universal enveloping algebra $U_{q} \mathfrak{s l}_{2}$. Also, for each $q$ the authors defined $q$ analogues of the weighted Bergman spaces $L_{a}^{2}\left(\left(1-|z|^{2}\right)^{\lambda} d \bar{z} d z\right)(\lambda \geq 0)$ on the unit disk and showed that $\mathcal{P}_{q, \lambda}$ could be realized as the algebra of Toeplitz operators (with symbols from $\mathcal{P}_{q, 0}$ ) on the $q$-weighted Bergman space corresponding to $\lambda$. This observation suggests that it is reasonable to regard $\mathcal{P}_{q, 0}$ as a genuine function algebra on the quantum unit disk and $\mathcal{P}_{q, \lambda}$ as a result of Toeplitz quantization of $\mathcal{P}_{q, 0}$ which, in addition, respects the $U_{q} \mathfrak{s l}_{2}$-actions in complete analogy with the classical setting.

This point of view was developed in details in [7]. There, in addition to the results of [6], q-analogues of covariant symbols and of the Berezin transform on the quantum unit disk was produced (see [8] for a concise account). Also, in [7] an explicit asymptotic formula, expressing the $q$-Berezin transform via a $q$-Laplace-Beltrami operator on the quantum disk, was derived. This allowed the authors to produce an analogue of the Berezin's star product and thus to obtain a formal deformation of the algebra $\mathcal{P}_{q, 0}$.

The present paper continues the study of the $q$-Berezin transform initiated in [7]. Here we investigate the case of the quantum unit ball, whose one-dimensional version is the aforementioned quantum unit disk, and generalize to this case almost all constructions of [7. Namely, we produce analogues of the weighted Bergman spaces, Toeplitz operators, covariant symbols, and the Berezin transform on the unit ball. Also, we define a $q$-analogue of the $S U(n, 1)$-invariant Laplace operator (the Laplace-Beltrami operator) and derive an explicit asymptotic formula expressing the $q$-Berezin transform via the $q$-Laplace-Beltrami operator.

It is appropriate to mention here that in the case of the quantum unit ball we encounter a new phenomenon imperceptible in the case of the quantum unit disk. Namely, there is no any satisfactory analogue of coherent states on the ball which are used to define covariant symbols of operators and regarded as a basic ingredient of the Berezin's theory. An appropriate analogue for the quantum unit disk was found in [7]. However, that was possible due to commutativity of the algebra of 'holomorphic functions' on the quantum disk, but the commutativity fails in the case of the quantum ball. Fortunately, even in the classical setting there is an alternative way to define covariant symbols. Namely, the map

$$
\begin{gathered}
\text { operator } \\
\text { on a weighted Bergman space }
\end{gathered} \quad \begin{gathered}
\text { covariant symbol, } \\
\text { a function on the ball }
\end{gathered}
$$

is, roughly speaking, the adjoint of the map

$$
\begin{gathered}
\text { function } \\
\text { on the ball }
\end{gathered} \text { on a weighted Bergman space }
$$


with respect to certain $S U(n, 1)$-invariant inner products in the spaces of functions and operators (see [5]). The significance of the Toeplitz and covariant calculi is of course well known and has been intensively studied. We exploit just this idea to define covariant symbols and thus the Berezin transform in the $q$-setting.

Let us turn now to description of the contents of the paper.

Our results rely heavily on function theory in the quantum unit ball. In Section 2 we recall the basic setup and results from that theory obtained earlier by L. Vaksman and his group [9, 10] in the more general setting of quantum matrix balls. In brief, we define an involutive algebra of 'polynomials' on the quantum $\mathbb{C}^{n}$ which generalize the algebra $\mathcal{P}_{q, 0}$ mentioned above. The algebra is endowed with an action of the quantum universal enveloping algebra $U_{q} \mathfrak{s u}_{n, 1}$. This is a counterpart of the classical $S U(n, 1)$-action on the unit ball. Using the polynomial algebra, we produce the spaces of 'finite functions' and 'distributions' on the quantum ball which inherit the $U_{q} \mathfrak{s u}_{n, 1}$-action. The crucial property, which justifies our definition of the space of finite functions on the quantum ball, is the existence of a $U_{q} \mathfrak{s u}_{n, 1}$-invariant integral on that space, an analogue of the integral with respect to the $S U(n, 1)$-invariant measure on the ball. We present an explicit formula for that integral. We note that the existence of the integral allows one to formulate many problems of harmonic analysis on the quantum ball. Though the present paper does not deal with harmonic analysis, we develop some partial results (see description of Section 4 below) which are needed to put our result in a coherent and rigorous mathematics context.

Section 3 is devoted to find an appropriate algebraic and analytical setting. There we start developing of the $q$-Berezin's theory and present all necessary definitions. First, we define analogues of the weighted Bergman spaces in the ball and produce the notion of Toeplitz operators with polynomial or finite symbols. Though the finite symbols are of more importance for our exposition, we devote a separate subsection to the study of the algebra of Toeplitz operators with polynomial symbols. This is motivated by the observation that this algebra is a multivariable generalization of the algebra $\mathcal{P}_{q, \lambda}$ studied in [6]. Results of [6] suggest that the algebra of Toeplitz operators with polynomial symbols might be interesting from the point of view of the theory of operator algebras. In the last subsection we present definitions of covariant symbols and of the $q$-Berezin transform. Thus, in Section 3 we define three important maps: the map that sends a function to the corresponding Toeplitz operator, the map that sends an operator to its covariant symbol (a function on the quantum ball), and, finally, their composition - the $q$-Berezin transform - which sends a function to the covariant symbol of the corresponding Toeplitz operator. We observe also that the three maps intertwine natural $U_{q} \mathfrak{s u}_{n, 1}$-actions on the spaces of functions and operators. This quantum group symmetry of the $q$-Berezin transform simplifies drastically many computations in subsequent sections.

To investigate the $q$-Berezin transform further, for instance, to find its asymptotic expansion, we need some results on harmonic analysis, particularly, on $q$-spherical transform in the quantum ball. These are presented in Section 4 . First of all, we define there an 
analogue of the Laplace-Beltrami operator on the ball. At first sight, our definition seems to be somewhat unusual: the $q$-Laplace-Beltrami operator appears as the first term in the asymptotic expansion of the $q$-Berezin transform. However, this agrees with the classical Berezin's theory. Besides, we believe that results of Section 4 provide a sufficient evidence of rationality of such a definition. In particular, a 'radial part' of our $q$-Laplace-Beltrami operator turns out to be a second order difference operator which tends to the radial part of the classical Laplace-Beltrami operator as $q$ approaches 1. Moreover, the difference operator turned out to be quite well studied by experts in the theory of $q$-special functions (see, for instance, [11]). In particular, the problem of expansion in eigenfunctions of that difference operator had being already solved, and we recall explicit formulas in the text. In view of our approach, those eigenfunctions should be regarded as analogues of the spherical functions on the ball while the expansion is an analogue of the spherical transform (see [12). Moreover, the Plancherel measure, which appears in the inverse transform, involves an analogue of the Harish-Chandra $c$-function for the ball.

After we present the results on the $q$-spherical transform, we turn back in Section 5 to the study of the $q$-Berezin transform. In the first subsection of Section 5 we consider its 'radial part'. We prove that the radial part of the transform is extended to a bounded selfadjoint operator on the space of square-integrable radial function on the quantum ball (we mean square-integrability with respect to the invariant $q$-integral). Moreover, the resulting operator commutes with the radial part of the $q$-Laplace-Beltrami operator which is also a bounded selfadjoint operator on the same space. Since the latter operator has simple spectrum (this is stated in Section 4), the radial part of the $q$-Berezin transform appears to be a function of the radial part of the $q$-Laplace-Beltrami operator. We found the function explicitly. We note that in the classical case the corresponding result was obtained in [5] for any Cartan domain. This computation has a number of consequences some of which we use further in Section 6. In the last subsection of Section 5, we present an asymptotic formula expressing the $q$-Berezin transform via the $q$-Laplace-Beltrami operator.

Section 6, the last section of the paper, is meant for those readers who are interested in various mathematical applications of the Berezin transform. It is however nevertheless related to quantization. In the theory of Wick quantization one associates to each function an operator so that the one-dimensional projections onto the coherent state at a point (in the phase space) is assigned to the delta function. However if the given Hilbert space has on orthogonal basis the projection onto the basis vectors are also natural subjects so it is interesting to compute their covariant symbols. Here in our setting we show that the spherical transforms of those covariant symbols are roughly speaking hypergeometric orthogonal $q$-polynomials. We derive orthogonality relations for those orthogonal polynomials, the so-called continuous dual $q$-Hahn polynomials [13]. The entire family of these polynomials depends on three parameters (not counting $q$ ) while we treat a two-parameter sub-family which appears naturally in connection with the $q$-Berezin transform. The idea 
we use appeared for the first time in [14] in the classical setting (see also [15]).

Let us make some comments about the most important agreements and notations used in the paper. First of all, the quantum-group parameter $q$ is supposed to be a number from the interval $(0,1)$. Next, we put $h:=\log q^{-2}$, so $h>0$; and $t:=q^{2 \alpha}$ will be the deformation parameter in the Berezin transform. Finally, we describe some convenient multi-index notations. We denote multi-indices by underlined small letters, the zero multi-index by $\underline{0}$. For $\underline{i}=\left(i_{1}, \ldots, i_{n}\right), \underline{j}=\left(j_{1}, \ldots, j_{n}\right)$ we put $|\underline{i}|=i_{1}+i_{2}+\ldots+i_{n}$ and $\underline{i} \times \underline{j}=\left(i_{1} \cdot j_{1}, \ldots, i_{n} \cdot j_{n}\right)$. For non-commuting variables $z_{1}, z_{2}, \ldots, z_{n}$ and $z_{1}^{*}, z_{2}^{*}, \ldots, z_{n}^{*}$ we set

$$
\mathbf{z}^{\underline{i}}:=z_{n}^{i_{n}} \cdot z_{n-1}^{i_{n-1}} \cdot \ldots \cdot z_{1}^{i_{1}}, \quad \mathbf{z}^{* i}:=z_{1}^{* i_{1}} \cdot \ldots \cdot z_{n-1}^{* i_{n-1}} \cdot z_{n}^{* i_{n}} .
$$

Finally, we denote $P(n)=\left\{\underline{k} \in \mathbb{Z}_{\geq 0}^{n} \mid k_{1} \geq k_{2} \geq \ldots \geq k_{n}\right\}$.

Acknowledgments. It is our pleasure to thank Leonid Vaksman for many interesting discussions. This research was supported by Royal Swedish Academy of Sciences under the program 'Cooperation between researchers in Sweden and the former Soviet Union'. We thank also the anonymous referee for expert comments and advices.

\section{Basics of function theory in the quantum unit ball}

The aim of Section 2 is to introduce some notions of function theory in the quantum ball. We define the involutive algebra of finite functions and the space of distributions on the $q$ ball. These spaces admit actions of certain quantum universal enveloping algebra. Finally, we produce an explicit formula for the invariant integral on the space of finite functions. The material presented in this section is not new: the quantum ball treated here is a particular case of the quantum matrix ball considered in details in [9, 10. Most results can be found in those papers, however, we reformulate some of them for our particular purpose.

\subsection{Polynomials on the quantum $\mathbb{C}^{n}$}

An initial object in constructing function theory in the quantum ball is the unital involutive algebra given by its generators $z_{1}, z_{2}, \ldots z_{n}$ and the relations

$$
\begin{gathered}
z_{i} z_{j}=q z_{j} z_{i}, \quad i<j \\
z_{i}^{*} z_{j}=q z_{j} z_{i}^{*}, \quad i \neq j \\
z_{j}^{*} z_{j}=q^{2} z_{j} z_{j}^{*}+\left(1-q^{2}\right)\left(1-\sum_{k=j+1}^{n} z_{k} z_{k}^{*}\right) .
\end{gathered}
$$


We denote this algebra by $\mathcal{P}\left(\mathbb{C}^{n}\right)_{q}$ and treat it as the polynomial algebra on the quantum $\mathbb{C}^{n}$. The algebra $\mathcal{P}\left(\mathbb{C}^{n}\right)_{q}$ is a particular case of the algebra $\operatorname{Pol}\left(\mathrm{Mat}_{m, n}\right)_{q}$ of polynomials on the quantum space of $m \times n$ matrices considered in [9, 10. Specifically, $\mathcal{P}\left(\mathbb{C}^{n}\right)_{q}$ coincides with $\operatorname{Pol}\left(\operatorname{Mat}_{1, n}\right)_{q}$. (Moreover, it is not hard to show that $\mathcal{P}\left(\mathbb{C}^{n}\right)_{q}$ is isomorphic to the known twisted CCR-algebra [16].)

In the sequel we use the algebra $\mathcal{P}\left(\mathbb{C}^{n}\right)_{q}$ to produce some 'functional' spaces and algebras on the quantum ball. Those spaces, as well as the algebra $\mathcal{P}\left(\mathbb{C}^{n}\right)_{q}$ itself, are endowed with structures of $U_{q} \mathfrak{s u}_{n, 1}$-modules where $U_{q} \mathfrak{s u}_{n, 1}$ is certain $*$-Hopf algebra called the quantized universal enveloping algebra for $\mathfrak{s u}_{n, 1}$. Let us turn to precise definitions.

We recall first the definition of $U_{q} \mathfrak{s u}_{n, 1}$. It is a 'real form' of the Drinfeld-Jimbo quantum universal enveloping algebra $U_{q} \mathfrak{s l}_{n+1}$. The latter algebra is the unital algebra given by the generators $E_{i}, F_{i}, K_{i}^{ \pm 1}, i=1,2, \ldots n$, and the following relations

$$
\begin{gathered}
K_{i} K_{j}=K_{j} K_{i}, \quad K_{i} K_{i}^{-1}=K_{i}^{-1} K_{i}=1, \quad K_{i} E_{j}=q^{a_{i j}} E_{j} K_{i}, \\
K_{i} F_{j}=q^{-a_{i j}} F_{j} K_{i}, \quad E_{i} F_{j}-F_{j} E_{i}=\delta_{i j}\left(K_{i}-K_{i}^{-1}\right) /\left(q-q^{-1}\right) \\
E_{i}^{2} E_{j}-\left(q+q^{-1}\right) E_{i} E_{j} E_{i}+E_{j} E_{i}^{2}=0, \quad|i-j|=1 \\
F_{i}^{2} F_{j}-\left(q+q^{-1}\right) F_{i} F_{j} F_{i}+F_{j} F_{i}^{2}=0, \quad|i-j|=1 \\
{\left[E_{i}, E_{j}\right]=\left[F_{i}, F_{j}\right]=0, \quad|i-j| \neq 1}
\end{gathered}
$$

with $\left(a_{i j}\right)$ being the Cartan matrix of type $A_{n}$. Moreover, $U_{q} \mathfrak{s l}_{n+1}$ is a Hopf algebra. The comultiplication $\Delta$, the antipode $S$, and the counit $\varepsilon$ are determined by

$$
\begin{gathered}
\Delta\left(E_{i}\right)=E_{i} \otimes 1+K_{i} \otimes E_{i}, \quad \Delta\left(F_{i}\right)=F_{i} \otimes K_{i}^{-1}+1 \otimes F_{i}, \quad \Delta\left(K_{i}\right)=K_{i} \otimes K_{i}, \\
S\left(E_{i}\right)=-K_{i}^{-1} E_{i}, \quad S\left(F_{i}\right)=-F_{i} K_{i}, \quad S\left(K_{i}\right)=K_{i}^{-1}, \\
\varepsilon\left(E_{i}\right)=\varepsilon\left(F_{i}\right)=0, \quad \varepsilon\left(K_{i}\right)=1 .
\end{gathered}
$$

The quantum universal enveloping algebra $U_{q} \mathfrak{s u}_{n, 1}$ is defined as the $*$-Hopf algebra $\left(U_{q} \mathfrak{s l}_{n+1}, *\right)$ with $*$ being the involution in $U_{q} \mathfrak{s l}_{n+1}$ given by

$$
\begin{array}{lll}
E_{n}^{*}=-K_{n} F_{n}, & F_{n}^{*}=-E_{n} K_{n}^{-1}, & \left(K_{n}^{ \pm 1}\right)^{*}=K_{n}^{ \pm 1}, \\
E_{j}^{*}=K_{j} F_{j}, & F_{j}^{*}=E_{j} K_{j}^{-1}, & \left(K_{j}^{ \pm 1}\right)^{*}=K_{j}^{ \pm 1}, \quad \text { for } \quad j \neq n .
\end{array}
$$

(see [17] for basic definitions concerning $*$-Hopf algebras).

We need also the notion of $U_{q} \mathfrak{s u}_{n, 1}$-module algebra. Let $A$ be a Hopf algebra. An algebra $F$ is said to be an $A$-module algebra if $F$ carries a structure of $A$-module and multiplication in $F$ agrees with the $A$-action, i.e. the multiplication $F \otimes F \rightarrow F$ is a morphism of $A$-modules. ${ }^{1}$ If $A$ or $F$ has some extra structures this definition includes natural additional requirements. For example, in the case of a unital algebra $F$ one adds

\footnotetext{
${ }^{1}$ We recall that for any $A$-modules $V_{1}, V_{2}$ their tensor product is endowed with an $A$-module structure via the comultiplication $\Delta: A \rightarrow A \otimes A$.
} 
the requirement of $A$-invariance of the unit: $\xi(1)=\varepsilon(\xi) \cdot 1, \xi \in A$. In the case of an involutive algebra $F$ and a $*$-Hopf algebra $A$ one imposes the requirement of agreement of the involutions:

$$
(\xi(f))^{*}=S(\xi)^{*}\left(f^{*}\right), \quad \xi \in A, f \in F .
$$

Some natural examples of module algebras appear in the classical setting. Suppose $X$ is a smooth $G$-space with $G$ being a Lie group. The $G$-action induces an action of the universal enveloping algebra $U \mathfrak{g}$ in the space $C^{\infty}(X)$ via differential operators. The usual Leibnitz rule for the differentiation of product of two functions means that $C^{\infty}(X)$ is a $U \mathfrak{g}$-module algebra. This example suggests the use of the language of module algebras for description of algebras of functions on quantum $G$-spaces.

The structure of $U_{q} \mathfrak{s u} u_{n, 1}$-module algebra in $\mathcal{P}\left(\mathbb{C}^{n}\right)_{q}$ which we are going to present has the following classical counterpart. The unit ball $U_{n}=\left\{\mathbf{z} \in \mathbb{C}^{n} \mid\|\mathbf{z}\|<1\right\}$ is a homogeneous space of the group $S U(n, 1)$ whose elements act via (biholomorphic) linearfractional transformations. Elements of the universal enveloping algebra $U \mathfrak{s u}_{n, 1}$ act on the space $C^{\infty}\left(U_{n}\right)$ via differential operators with polynomial coefficients and thus keep the subspace of polynomials invariant. This induces a $U \mathfrak{s u}{ }_{n, 1}$-module algebra structure in the polynomial algebra on $\mathbb{C}^{n}$. We turn now to the quantum case.

To describe the $U_{q} \mathfrak{s u}_{n, 1}$-module algebra structure in $\mathcal{P}\left(\mathbb{C}^{n}\right)_{q}$ we consider the action of the elements $E_{i}, F_{i}, K_{i}^{ \pm 1}$ on the generators $z_{i}, z_{i}^{*}$. Moreover, by (2.4) it is sufficient to present formulas for the action of $E_{i}, F_{i}, K_{i}^{ \pm 1}$ on the 'holomorphic' part $z_{1}, \ldots z_{n}$ of generators of $\mathcal{P}\left(\mathbb{C}^{n}\right)_{q}$. These are given in the following

Proposition 2.1 There exists a unique structure of $U_{q} \mathfrak{s u} \mathfrak{n}_{n, 1}$-module algebra in $\mathcal{P}\left(\mathbb{C}^{n}\right)_{q}$ such that, for $k \neq n$,

$$
\begin{gathered}
K_{k} z_{i}=\left\{\begin{array}{cc}
q z_{i}, & i=k \\
q^{-1} z_{i}, & i=k+1 \\
z_{i}, & \text { otherwise }
\end{array}\right. \\
F_{k} z_{i}=q^{1 / 2} \cdot\left\{\begin{array}{cc}
z_{i+1}, & i=k \\
0, & \text { otherwise }
\end{array}\right. \\
E_{k} z_{i}=q^{-1 / 2} \cdot\left\{\begin{array}{cc}
z_{i-1}, & i=k+1 \\
0, & \text { otherwise }
\end{array}\right.
\end{gathered}
$$

and

$$
\begin{gathered}
K_{n} z_{i}=\left\{\begin{array}{cc}
q^{2} z_{i}, & i=n \\
q z_{i}, & \text { otherwise, }
\end{array}\right. \\
F_{n} z_{i}=q^{1 / 2} \cdot\left\{\begin{array}{cc}
1, & i=n \\
0, & \text { otherwise },
\end{array}\right. \\
E_{n} z_{i}=-q^{1 / 2} \cdot\left\{\begin{array}{cc}
z_{n}^{2}, & i=n \\
z_{n} z_{i}, & \text { otherwise. }
\end{array}\right.
\end{gathered}
$$


This statement may be deduced from Proposition 2.1 and Corollary 5.6 in 9] by easy computations. In the next subsection we will introduce some other important $U_{q \mathfrak{s u}} \mathfrak{1}^{-}$ module algebras.

\subsection{Finite functions on the quantum ball}

It should be noted that in the classical case the space of polynomials does not suit purposes of harmonic analysis in the ball because the volume of the ball with respect to the $S U(n, 1)$-invariant measure

$$
d \nu(\mathbf{z})=\frac{d m(\mathbf{z})}{\left(1-\|\mathbf{z}\|^{2}\right)^{n+1}}
$$

(where $d m(\mathbf{z})$ is the normalized Lebesgue measure) is infinite. One observes the same problem in the quantum setting: there is no $U_{q} \mathfrak{s} \mathfrak{u}_{n, 1}$-invariant integral on $\mathcal{P}\left(\mathbb{C}^{n}\right)_{q}$, i. e. a linear positive functional $\nu: \mathcal{P}\left(\mathbb{C}^{n}\right)_{q} \rightarrow \mathbb{C}$ such that $\nu(\xi(f))=\varepsilon(\xi) \cdot \nu(f)$ for any $\xi \in U_{q} \mathfrak{s u}_{n, 1}$ ( $\varepsilon$ is the counit of $U_{q} \mathfrak{s u _ { n , 1 }}$ ). Thus it is reasonable to look for a quantum counterpart of the space of finite functions on the ball (functions with compact supports inside the ball). The following construction for such a counterpart was proposed in 9, Section 7].

Let us add to the algebra $\mathcal{P}\left(\mathbb{C}^{n}\right)_{q}$ one more generator $f_{0}$ which satisfies the relations

$$
f_{0}^{*}=f_{0}^{2}=f_{0}, \quad z_{i}^{*} f_{0}=f_{0} z_{i}=0, \quad i=1,2, \ldots n .
$$

The resulting involutive algebra will be denoted by $\mathcal{F}\left(U_{n}\right)_{q}$. It is demonstrated in 9, Section 7] that one may extend the structure of $U_{q} \mathfrak{s u}_{n, 1}$-module algebra in $\mathcal{P}\left(\mathbb{C}^{n}\right)_{q}$ to one in $\mathcal{F}\left(U_{n}\right)_{q}$ as follows

$$
K_{k} f_{0}=f_{0}, \quad F_{k} f_{0}=E_{k} f_{0}=0
$$

with $k \neq n$ and

$$
K_{n} f_{0}=f_{0}, \quad F_{n} f_{0}=-\frac{q^{1 / 2}}{q^{-2}-1} f_{0} \cdot z_{n}^{*}, \quad E_{n} f_{0}=-\frac{q^{1 / 2}}{1-q^{2}} z_{n} \cdot f_{0} .
$$

The involutive algebra $\mathcal{D}\left(U_{n}\right)_{q}$ of finite functions on the quantum ball is defined as the two-sided ideal $\mathcal{F}\left(U_{n}\right)_{q} \cdot f_{0} \cdot \mathcal{F}\left(U_{n}\right)_{q}$ in $\mathcal{F}\left(U_{n}\right)_{q}$. Due to (2.13), (2.14), $\mathcal{D}\left(U_{n}\right)_{q}$ is a $U_{q} \mathfrak{s u} u_{n, 1^{-}}$ module subalgebra in $\mathcal{F}\left(U_{n}\right)_{q}$. In the next subsection we present an explicit formula for a $U_{q} \mathfrak{s u} \mathfrak{u}_{n, 1}$-invariant integral on $\mathcal{D}\left(U_{n}\right)_{q}$.

The above definition of $\mathcal{D}\left(U_{n}\right)_{q}$ is convenient for many purposes but performing computations. We present therefore an alternative description of $\mathcal{D}\left(U_{n}\right)_{q}$. For that purpose we let $H$ be the $\mathcal{P}\left(\mathbb{C}^{n}\right)_{q}$-module given by its unique generator $e_{0}$ such that $z_{i}^{*} e_{0}=0$, $i=1,2, \ldots n$. By the formulas (2.2) and (2.3),$H=\mathcal{P}\left(\mathbb{C}^{n}\right)_{q} \cdot e_{0}=\mathbb{C}\left[\mathbb{C}^{n}\right]_{q} \cdot e_{0}$ with $\mathbb{C}\left[\mathbb{C}^{n}\right]_{q}$ being the unital (non-involutive) subalgebra in $\mathcal{P}\left(\mathbb{C}^{n}\right)_{q}$ generated by $z_{i}, i=1,2, \ldots n$. Moreover, the elements $\left\{\mathbf{z}^{\underline{m}} \cdot e_{0}\right\}_{\underline{m} \in \mathbb{Z}_{\geq 0}^{n}}$ constitute a basis in $H$. The following statement may be deduced from known results concerning the twisted CCR-algebra (see, for instance, [18]. 
Proposition 2.2 i) There exists a (unique up to a positive multiplier) scalar product in $H$ such that

$$
\left(f \cdot e_{1}, e_{2}\right)=\left(e_{1}, f^{*} \cdot e_{2}\right)
$$

for any $f \in \mathcal{P}\left(\mathbb{C}^{n}\right)_{q}$ and $e_{1}, e_{2} \in H$.

ii) The corresponding *-representation $T$ of $\mathcal{P}\left(\mathbb{C}^{n}\right)_{q}$ in the completion $\bar{H}$ of the preHilbert space $H$ (the so-called Fock representation) is a faithful irreducible representation by bounded operators.

We let $C^{*}\left(\mathcal{P}\left(\mathbb{C}^{n}\right)_{q}\right)$ be the $C^{*}$-algebra generated by $\mathcal{P}\left(\mathbb{C}^{n}\right)_{q}$ via the representation $T$. We describe now an embedding of $\mathcal{D}\left(U_{n}\right)_{q}$ in $C^{*}\left(\mathcal{P}\left(\mathbb{C}^{n}\right)_{q}\right)$.

The representation $T$ can be extended to a faithful *-representation of the algebra $\mathcal{F}\left(U_{n}\right)_{q}$ by setting

$$
T\left(f_{0}\right)=\text { orthogonal projection onto } \mathbb{C} \cdot e_{0} .
$$

It is easy to show that the subalgebra $\left\{T(f) \mid f \in \mathcal{D}\left(U_{n}\right)_{q}\right\}$ of the algebra of bounded operators on $\bar{H}$ coincides with the subalgebra of those operators whose matrices in the basis $\left\{\mathbf{z}^{\underline{m}} \cdot e_{0}\right\}_{\underline{m} \in \mathbb{Z}_{\geq 0}^{n}}$ have only finitely many non-zero entries. Thus the algebra $\mathcal{D}\left(U_{n}\right)_{q}$ can be realized as a subalgebra of $C^{*}\left(\mathcal{P}\left(\mathbb{C}^{n}\right)_{q}\right)$. It admits the following transparent description.

Let $y_{i}=1-z_{i} z_{i}^{*}-\ldots-z_{n} z_{n}^{*} \in \mathcal{P}\left(\mathbb{C}^{n}\right)_{q}, i=1,2, \ldots n$. Then $\left(y_{1}, \ldots, y_{n}\right)$ is a tuple of pairwise commuting positive operators on $\bar{H}$. The joint spectrum of the tuple $\left(y_{1}, \ldots, y_{n}\right)$ in $C^{*}\left(\mathcal{P}\left(\mathbb{C}^{n}\right)_{q}\right)$ is the closure in $\mathbb{R}^{n}$ of the ' $q$-simplex'

$$
\mathfrak{M}=\left\{\left(q^{2 k_{1}}, q^{2 k_{2}}, \ldots q^{2 k_{n}}\right) \mid \underline{k} \in P(n)\right\}
$$

$(P(n)$ is defined in the Introduction). This is a consequence of the commutation relations

$$
z_{i} y_{j}=\left\{\begin{array}{cc}
q^{-2} y_{j} z_{i}, & i \leq j \\
y_{j} z_{i}, & \text { otherwise, }
\end{array} \quad z_{i}^{*} y_{j}=\left\{\begin{array}{cc}
q^{2} y_{j} z_{i}^{*}, & i \leq j \\
y_{j} z_{i}^{*}, & \text { otherwise. }
\end{array}\right.\right.
$$

We will hence forth identify a function $f\left(y_{1}, \ldots, y_{n}\right)$ with a function $f$ on the set $\mathfrak{M}$ via the spectral calculus.

Using the definition of $y_{i}$ 's and (2.16), one can write an arbitrary element $f \in \mathcal{P}\left(\mathbb{C}^{n}\right)_{q}$ uniquely in the form

$$
f=\sum_{\underline{i} \times \underline{j}=0} \mathbf{z}^{\underline{i}} f_{\underline{i}, \underline{j}}\left(y_{1}, y_{2}, \ldots y_{n}\right) \mathbf{z}^{* \underline{j}} .
$$

The subalgebra $\mathcal{D}\left(U_{n}\right)_{q} \subset C^{*}\left(\mathcal{P}\left(\mathbb{C}^{n}\right)_{q}\right)$ may be identified with the algebra of finite sums of the form (2.17) whose coefficients are functions on $\mathfrak{M}$ with finite supports.

We will use the both descriptions of $\mathcal{D}\left(U_{n}\right)_{q}$. It is clear that the 'distinguished' finite function $f_{0}$ which appears in the definition of $\mathcal{D}\left(U_{n}\right)_{q}$ may be described, in those terms, as follows: $f_{\underline{i}, \underline{j}} \equiv 0$ provided $\underline{i} \neq \underline{0}$ or $\underline{j} \neq \underline{0}$, and

$$
f_{\underline{0}, \underline{0}}\left(q^{2 k_{1}}, q^{2 k_{2}}, \ldots q^{2 k_{n}}\right)=\left\{\begin{array}{l}
1, \quad \underline{k}=\underline{0} \\
0, \quad \text { otherwise. }
\end{array}\right.
$$

The following crucial property of $f_{0}$ will simplify proofs of many results. 
Proposition $2.3 f_{0}$ generates $\mathcal{D}\left(U_{n}\right)_{q}$ as a $U_{q} \mathfrak{s u}_{n, 1}$-module.

A proof can be found in [9, Section 7].

\subsection{Invariant integral}

In this subsection we present an explicit formula for the $U_{q} \mathfrak{s u}_{n, 1}$-invariant integral on the quantum ball. This formula was found in [9, Section 9].

Remind the notation $T$ for the Fock representation of $\mathcal{P}\left(\mathbb{C}^{n}\right)_{q}$ and $H$ for a $\mathcal{P}\left(\mathbb{C}^{n}\right)_{q^{-}}$ module, a dense linear subspace in the Hilbert space $\bar{H}$ of th representation $T$ (see the previous subsection). It is easy to observe that $T(f)(H) \subset H$ for any finite function $f$. Thus $H$ become a $\mathcal{D}\left(U_{n}\right)_{q}$-module. It is convenient to identify the $\mathcal{D}\left(U_{n}\right)_{q}$-module $H$ with the left ideal $\mathbb{C}\left[\mathbb{C}^{n}\right]_{q} \cdot f_{0}$ (this is possible due to (2.12) ), with which we may equip $H$ with some extra structures. In particular, the isomorphism of $\mathcal{D}\left(U_{n}\right)_{q}$-modules

$$
H \simeq \mathbb{C}\left[\mathbb{C}^{n}\right]_{q} \cdot f_{0} \subset \mathcal{D}\left(U_{n}\right)_{q}
$$

and formulas (2.5), (2.8), (2.13), (2.14) define $H$ as a $U_{q} \mathfrak{h}$-module where $U_{q} \mathfrak{h}$ is the Hopf subalgebra in $U_{q} \mathfrak{s l}_{n+1}$ generated by $K_{i}^{ \pm 1}, i=1,2, \ldots n$. Denote the corresponding representation of $U_{q} \mathfrak{h}$ in $H$ by $\Gamma$. The following statement was proved in [9].

Proposition 2.4 The linear functional on $\mathcal{D}\left(U_{n}\right)_{q}$ given by

$$
f \mapsto \nu(f)=\operatorname{tr}\left(T(f) \Gamma\left(\prod_{j=1}^{n} K_{j}^{-j(n+1-j)}\right)\right)
$$

is a $U_{q} \mathfrak{s u}_{n, 1}$-invariant integral, i.e. $\nu\left(f^{*} f\right)>0, f \neq 0$, and $\nu(\xi(f))=\varepsilon(\xi) \cdot \nu(f)$, $\xi \in U_{q} \mathfrak{s u}_{n, 1}$.

We shall use the normalized integral

$$
\int_{U_{n}} f d \nu_{q}=\left(q^{2} ; q^{2}\right)_{n} \cdot \nu(f)
$$

with $\left(q^{2} ; q^{2}\right)_{n}=\left(1-q^{2}\right)\left(1-q^{4}\right) \ldots\left(1-q^{2 n}\right)$.

The invariant integral is unique up to a positive scalar. This is an immediate consequence of Proposition 2.3 .

We can rewrite now the formula for the invariant integral in a more convenient form. Suppose $f \in \mathcal{D}\left(U_{n}\right)_{q}$ is written in the form (2.17). Then one checks easily that

$$
\int_{U_{n}} f d \nu_{q}=\left(q^{2} ; q^{2}\right)_{n} \cdot \sum_{\underline{k} \in P(n)} f_{\underline{0}, \underline{0}}\left(q^{2 k_{1}}, q^{2 k_{2}}, \ldots q^{2 k_{n}}\right) \cdot q^{-2 n k_{1}} \cdot q^{2 k_{2}} \cdot \ldots \cdot q^{2 k_{n}} .
$$

In the next proposition we explain how the $U_{q} \mathfrak{s u} \mathfrak{u}_{n, 1}$-invariant integral makes $\mathcal{D}\left(U_{n}\right)_{q}$ into a unitary $U_{q} \mathfrak{s u}_{n, 1}$-module and thus provides a setup for harmonic analysis in the quantum ball. 
Proposition 2.5 The scalar product in $\mathcal{D}\left(U_{n}\right)_{q}$ given by

$$
\left(\varphi_{1}, \varphi_{2}\right)=\int_{U_{n}} \varphi_{2}^{*} \cdot \varphi_{1} d \nu_{q}
$$

is $U_{q} \mathfrak{s u}_{n, 1}$-invariant, i.e. $\left(\xi\left(\varphi_{1}\right), \varphi_{2}\right)=\left(\varphi_{1}, \xi^{*}\left(\varphi_{2}\right)\right)$ for any $\varphi_{1}, \varphi_{2} \in \mathcal{D}\left(U_{n}\right)_{q}$ and any $\xi \in U_{q} \mathfrak{s u}_{n, 1}$.

Proof. This follows from the condition (2.4) in $\mathcal{D}\left(U_{n}\right)_{q}$ and the following formula of 'integrating by parts': for any $\varphi_{1}, \varphi_{2} \in \mathcal{D}\left(U_{n}\right)_{q}$ and $\xi \in U_{q} \mathfrak{s u}_{n, 1}$ one has

$$
\int_{U_{n}} \xi\left(\varphi_{1}\right) \cdot \varphi_{2} d \nu_{q}=\int_{U_{n}} \varphi_{1} \cdot S(\xi)\left(\varphi_{2}\right) d \nu_{q}
$$

with $S$ being the antipode of $U_{q} \mathfrak{s u}_{n, 1}$. To prove the latter formula, it is sufficient to verify the equality on generators $E_{i}, F_{i}, K_{i}^{ \pm 1}$ of $U_{q} \mathfrak{s u}_{n, 1}$. In this particular case the equality is equivalent to the $U_{q} \mathfrak{s u}_{n, 1}$-invariance of the integral.

\subsection{Distributions on the quantum ball}

The aim of this subsection is to define the space $\mathcal{D}\left(U_{n}\right)_{q}^{\prime}$ of distributions on the quantum ball. We follow ideas in [19, Section 1] where the simplest case $n=1$ is treated.

Equip the vector space $\mathcal{D}\left(U_{n}\right)_{q}$ with the weakest topology so that all the linear functionals

$$
l_{\underline{i}, \underline{j}}^{\underline{k}}: f \mapsto f_{\underline{i, j}, \underline{j}}\left(q^{2 k_{1}}, q^{2 k_{2}}, \ldots q^{2 k_{n}}\right)
$$

are continuous (here $\underline{i}, \underline{j} \in \mathbb{Z}_{\geq 0}^{n}$ satisfy $\underline{i} \times \underline{j}=\underline{0}, \underline{k} \in P(n)$ ), and $f_{\underline{i}, \underline{j}}$ is the corresponding coefficient in the expansion (2.17) of $f$ ).

The following results can be proved by direct however tedious computations, which we omit.

Proposition 2.6 For a fixed $f \in \mathcal{D}\left(U_{n}\right)_{q}$ the operator in $\mathcal{D}\left(U_{n}\right)_{q}$ of the right multiplication by $f$ is continuous. Also, the $U_{q} \mathfrak{s u}_{n, 1}$-action in $\mathcal{D}\left(U_{n}\right)_{q}$ is continuous.

Let $\mathcal{D}\left(U_{n}\right)_{q}^{\prime}$ be the completion of the topological vector space $\mathcal{D}\left(U_{n}\right)_{q}$. We shall use the concrete realization of $\mathcal{D}\left(U_{n}\right)_{q}^{\prime}$ as the space of infinite sums of the form (2.17) whose coefficients $f_{\underline{\underline{j}}, \underline{j}}$ are arbitrary functions on the $q$-simplex $\mathfrak{M}$ (see (2.15)). The above proposition, by continuity, implies that $\mathcal{D}\left(U_{n}\right)_{q}^{\prime}$ is a right $\mathcal{D}\left(U_{n}\right)_{q}$-module and a $U_{q} \mathfrak{s u}_{n, 1}$-module. Moreover, these structures agree: the multiplication map $\mathcal{D}\left(U_{n}\right)_{q}^{\prime} \otimes \mathcal{D}\left(U_{n}\right)_{q} \rightarrow \mathcal{D}\left(U_{n}\right)_{q}^{\prime}$ is a morphism of $U_{q} \mathfrak{s u}_{n, 1}$-modules. This is a consequence of $U_{q} \mathfrak{s u}_{n, 1}$-moduleness of the algebra $\mathcal{D}\left(U_{n}\right)_{q}$.

Let us construct a non-degenerate pairing $\mathcal{D}\left(U_{n}\right)_{q}^{\prime} \times \mathcal{D}\left(U_{n}\right)_{q} \rightarrow \mathbb{C}$ which will justify the term 'distribution'. Let $\left\{\psi_{\beta}\right\}_{\beta}$ be a convergent net in $\mathcal{D}\left(U_{n}\right)_{q}$ and suppose $\lim _{\beta} \psi_{\beta}=\psi$, $\psi \in \mathcal{D}\left(U_{n}\right)_{q}^{\prime}$. Then for any $\varphi \in \mathcal{D}\left(U_{n}\right)_{q}$ the $\operatorname{limit} \lim _{\beta} \int_{U_{n}} \psi_{\beta} \varphi d \nu_{q}$ exists and depends 
only on $\psi$ and $\varphi$ (i. e. independent of a choice of $\psi_{\beta}$ ). Indeed, let us prove existence of $\lim \int_{U_{n}} \psi_{\beta} \varphi d \nu_{q}$. Proposition 2.3 implies that there exists $\xi_{\varphi} \in U_{q} \mathfrak{s u}_{n, 1}$ such that $\varphi=$ $\xi_{\varphi}\left(f_{0}\right)$. By (2.19)

$$
\int_{U_{n}} \psi_{\alpha} \varphi d \nu_{q}=\int_{U_{n}} S^{-1}\left(\xi_{\varphi}\right)\left(\psi_{\beta}\right) f_{0} d \nu_{q}
$$

Since the $U_{q} \mathfrak{s u}_{n, 1}$-action is continuous, it is sufficient now to prove existence of the limit in the particular case $\varphi:=f_{0}$. But in this case existence equivalent to continuity of the functional $l \underline{\underline{0}} \underline{0}$ and thus is obvious. Independence of the limit on a particular choice of the net $\left\{\psi_{\beta}\right\}$ can be proved by the same arguments.

Thus we get a well defined pairing

$$
\mathcal{D}\left(U_{n}\right)_{q}^{\prime} \times \mathcal{D}\left(U_{n}\right)_{q} \rightarrow \mathbb{C}, \quad(\psi, \varphi) \mapsto \int_{U_{n}} \psi \varphi d \nu_{q}
$$

and due to (2.19), it satisfies the property

$$
\int_{U_{n}} \xi(\psi) \cdot \varphi d \nu_{q}=\int_{U_{n}} \psi \cdot S(\xi)(\varphi) d \nu_{q}
$$

for any $\psi \in \mathcal{D}\left(U_{n}\right)_{q}^{\prime}, \varphi \in \mathcal{D}\left(U_{n}\right)_{q}$, and $\xi \in U_{q} \mathfrak{s u} u_{n, 1}$. In other words, we have constructed a morphism of $U_{q} \mathfrak{s u}_{n, 1}$-modules ${ }^{2}$

$$
\mathcal{D}\left(U_{n}\right)_{q}^{\prime} \rightarrow \text { dual of } \mathcal{D}\left(U_{n}\right)_{q}
$$

In fact, this is an isomorphism. This can be proved by computation just as it was done in [19, Section 1] in the case $n=1$.

\section{$3 q$-Berezin transform}

The aim of this section is to construct a $q$-analogue of the Berezin transform in the unit ball. For that purpose we define also q-analogues of the weighted Bergman spaces, Toeplitz operators, and covariant symbols.

\section{$3.1 q$-Weighted Bergman spaces}

In the classical case the weighted Bergman space is defined as the closure of the space of holomorphic polynomials with respect to the norm

$$
\|f\|_{\alpha}=\left(\frac{\Gamma(n+\alpha+1)}{\Gamma(n+1) \Gamma(\alpha+1)} \int_{U_{n}}|f(\mathbf{z})|^{2}\left(1-\|\mathbf{z}\|^{2}\right)^{\alpha} d m(\mathbf{z})\right)^{\frac{1}{2}}
$$

\footnotetext{
${ }^{2}$ For an $A$-modules $V$ the dual space $V^{*}$ is endowed with an $A$-module structure via the antipode: $\xi f(\cdot)=f(S(\xi) \cdot)$.
} 
Here $\alpha>-1, d m$ is the normalized Lebesgue measure, and the multiplier $\frac{\Gamma(n+\alpha+1)}{\Gamma(n+1) \Gamma(\alpha+1)}$ normalizes the measure $d m_{\alpha}=\left(1-\|\mathbf{z}\|^{2}\right)^{\alpha} d m(\mathbf{z})$. Let us define a $q$-analogue of $d m_{\alpha}$.

Suppose $\alpha \in \mathbb{Z}_{\geq 0}$. The formula (3.1), along with (2.11), suggests the following definition of the $q$-weighted integral

$$
\int_{U_{n}} f d m_{\alpha, q}=\frac{\Gamma_{q^{2}}(n+\alpha+1)}{\Gamma_{q^{2}}(n+1) \Gamma_{q^{2}}(\alpha+1)} \int_{U_{n}} f \cdot y_{1}^{\alpha-n-1} d \nu_{q}
$$

with $y_{1}=1-z_{1} z_{1}^{*}-\ldots-z_{n} z_{n}^{*} \in \mathcal{P}\left(\mathbb{C}^{n}\right)_{q}$ (see subsection 2.2). Here we use the standard notation [20]:

with

$$
\Gamma_{q}(x)=\frac{(q ; q)_{\infty}}{\left(q^{x} ; q\right)_{\infty}}(1-q)^{1-x}
$$

$$
(a ; q)_{\infty}=(1-a)(1-a q)\left(1-a q^{2}\right) \ldots, \quad(a ; q)_{\gamma}=\frac{(a ; q)_{\infty}}{\left(a q^{\gamma} ; q\right)_{\infty}}, \quad \gamma \in \mathbb{C} .
$$

Using (2.18) and the definition of $\Gamma_{q^{2}}$, we may rewrite the integral in the following way

$$
\int_{U_{n}} f d m_{\alpha, q}=\left(q^{2 \alpha+2} ; q^{2}\right)_{n} \sum_{\underline{k} \in P(n)} f_{\underline{0}, \underline{0}}\left(q^{2 k_{1}}, q^{2 k_{2}}, \ldots q^{2 k_{n}}\right) \cdot q^{2 k_{1}(\alpha+1)} \cdot q^{2 k_{2}} \cdot \ldots \cdot q^{2 k_{n}} .
$$

The latter formula can be used to define the $q$-weighted integral for arbitrary $\alpha \in \mathbb{R}$.

It is reasonable to define $L^{2}\left(d m_{\alpha, q}\right)$ as the completion of $\mathcal{D}\left(U_{n}\right)_{q}$ with respect to the norm

$$
\|f\|_{\alpha, q}=\left(\int_{U_{n}} f^{*} \cdot f d m_{\alpha, q}\right)^{\frac{1}{2}} .
$$

We shall use the concrete realization of $L^{2}\left(d m_{\alpha, q}\right)$ as the subspace in $\mathcal{D}\left(U_{n}\right)_{q}^{\prime}$ of those distributions for which the right-hand side is finite.

From now on we suppose $\alpha$ is a positive real number. In this case, we have $\mathcal{P}\left(\mathbb{C}^{n}\right)_{q} \subset$ $L^{2}\left(d m_{\alpha, q}\right)$ (this may be checked by direct computations using formula (3.3) ). By analogy with the classical case, we define the $q$-weighted Bergman space $L_{a}^{2}\left(d m_{\alpha, q}\right)$ as the closure in $L^{2}\left(d m_{\alpha, q}\right)$ of the subspace $\mathbb{C}\left[\mathbb{C}^{n}\right]_{q}$ of 'holomorphic' polynomials.

Proposition 3.1 The monomials $\mathbf{z}^{\underline{m}}$ constitute an orthogonal basis in $L_{a}^{2}\left(d m_{\alpha, q}\right)$ and

$$
\left\|\mathbf{z}^{\underline{m}}\right\|_{\alpha, q}^{2}=\frac{\Gamma_{q^{2}}(n+\alpha+1) \Gamma_{q^{2}}\left(m_{1}+1\right) \ldots \Gamma_{q^{2}}\left(m_{n}+1\right)}{\Gamma_{q^{2}}\left(m_{1}+\ldots+m_{n}+n+\alpha+1\right)} .
$$

Proof. By the above definitions

$$
\left(\mathbf{z}^{\underline{m}}, \mathbf{z}^{\underline{\underline{l}}}\right)_{\alpha, q}=\left(q^{2 \alpha+2} ; q^{2}\right)_{n} \sum_{\underline{k} \in P(n)} f_{\underline{0}, \underline{0}}\left(q^{2 k_{1}}, q^{2 k_{2}}, \ldots q^{2 k_{n}}\right) \cdot q^{2 k_{1}(\alpha+1)} \cdot q^{2 k_{2}} \cdot \ldots \cdot q^{2 k_{n}},
$$

where $f_{\underline{0}, \underline{0}}$ is the corresponding term in the expansion (2.17) of the polynomial $\mathbf{z}^{* \underline{k}} \mathbf{z}^{\underline{m}}$. Obviously, the $f_{\underline{0}, \underline{0}}$-term vanishes for $\underline{m} \neq \underline{l}$. This implies the pairwise orthogonality of the monomials. Suppose $\underline{m}=\underline{l}$. It is easy to show that

$$
\mathbf{z}^{* \underline{m}} \mathbf{z}^{\underline{m}}=y_{2}^{m_{1}} y_{3}^{m_{2}} \ldots y_{n}^{m_{n-1}}\left(q^{2} \frac{y_{1}}{y_{2}} ; q^{2}\right)_{m_{1}}\left(q^{2} \frac{y_{2}}{y_{3}} ; q^{2}\right)_{m_{2}} \ldots\left(q^{2} \frac{y_{n-1}}{y_{n}} ; q^{2}\right)_{m_{n-1}}\left(q^{2} y_{n} ; q^{2}\right)_{m_{n}} .
$$


Denote by $f\left(y_{1}, y_{2}, \ldots y_{n}\right)$ the right-hand side of the latter equality. One has

$\sum_{\underline{k} \in P(n)} f\left(q^{2 k_{1}}, q^{2 k_{2}}, \ldots q^{2 k_{n}}\right) \cdot q^{2 k_{1}(\alpha+1)+2 k_{2}+\ldots+2 k_{n}}=\frac{1}{\left(1-q^{2}\right)^{n}} \prod_{j=0}^{n-1} \int_{0}^{1} t^{\alpha+m_{1}+\ldots+m_{j}+j}\left(q^{2} t ; q^{2}\right)_{m_{j+1}} d_{q^{2}} t$

where $\int_{0}^{1} f(t) d_{q^{2}} t$ is the Jackson integral given by

$$
\int_{0}^{1} f(t) d_{q^{2}} t=\left(1-q^{2}\right) \sum_{l=0}^{\infty} f\left(q^{2 l}\right) q^{2 l}
$$

What remains is to use the following well known formula [20]:

$$
\int_{0}^{1} t^{a}\left(q^{2} t ; q^{2}\right)_{b} d_{q^{2}} t=\frac{\Gamma_{q^{2}}(a+1) \Gamma_{q^{2}}(b+1)}{\Gamma_{q^{2}}(a+b+2)} .
$$

In the classical case the Hilbert space $L^{2}\left(d m_{\alpha}\right)$ admits a natural unitary $\widetilde{S U(n, 1)}$ action, where $\widehat{S U(n, 1)}$ is the universal covering of $S U(n, 1)$. The invariant subspace $L_{a}^{2}\left(d m_{\alpha}\right)$ of holomorphic functions is called the representation of the holomorphic discrete series [21], 222]. The corresponding infinitesimal $U \mathfrak{s u}_{n, 1}$-action is obtained by a simple twisting of the natural action. Below, we produce an analogue of the twisted $U \mathfrak{s u} \mathfrak{u}_{n, 1^{-}}$ action.

It was proved in [10, Section 6] that there exists a unique representation $\pi_{\alpha}$ of $U_{q} \mathfrak{s u} \mathfrak{u}_{n, 1}$ in $\mathcal{P}\left(\mathbb{C}^{n}\right)_{q}$ and $\mathcal{D}\left(U_{n}\right)_{q}$ such that for all $f \in \mathcal{P}\left(\mathbb{C}^{n}\right)_{q}\left(\right.$ or $\left.f \in \mathcal{D}\left(U_{n}\right)_{q}\right)$

$$
\begin{gathered}
\pi_{\alpha}\left(E_{j}\right) f= \begin{cases}E_{j} f, & j \neq n, \\
E_{n} f-q^{1 / 2} \frac{1-q^{2 \alpha+2 n+2}}{1-q^{2}}\left(K_{n} f\right) z_{n}, & j=n,\end{cases} \\
\pi_{\alpha}\left(F_{j}\right) f= \begin{cases}F_{j} f, & j \neq n, \\
q^{-\alpha-n-1} F_{n} f, & j=n,\end{cases} \\
\pi_{\alpha}\left(K_{j}^{ \pm 1}\right) f= \begin{cases}K_{j}^{ \pm 1} f, & j \neq n, \\
q^{ \pm(\alpha+n+1)} K_{n}^{ \pm 1} f, & j=n,\end{cases}
\end{gathered}
$$

where $E_{j} f, F_{j} f, K_{j} f$ are defined in Proposition 2.1 $(\underline{(2.13)})$ and (2.14) for $\left.f \in \mathcal{D}\left(U_{n}\right)_{q}\right)$. In order to not consider the polynomials and finite functions separately, it will be convenient sometimes to regard $\pi_{\alpha}$ as a representation of $U_{q} \mathfrak{s u} u_{n, 1}$ in $\mathcal{F}\left(U_{n}\right)_{q}$.

The following statement is proved in [10, Section 6].

Proposition 3.2 For any $\varphi_{1}, \varphi_{2} \in \mathcal{F}\left(U_{n}\right)_{q}$ and any $\xi \in U_{q} \mathfrak{s u}_{n, 1}$

$$
\left(\pi_{\alpha}(\xi) \varphi_{1}, \varphi_{2}\right)_{\alpha, q}=\left(\varphi_{1}, \pi_{\alpha}\left(\xi^{*}\right) \varphi_{2}\right)_{\alpha, q}
$$

with $(\cdot, \cdot)_{\alpha, q}$ being the scalar product in $L^{2}\left(d m_{\alpha, q}\right)$. 
Note that the subspace $\mathbb{C}\left[\mathbb{C}^{n}\right]_{q} \subset \mathcal{F}\left(U_{n}\right)_{q}$ is actually a $U_{q} \mathfrak{s u} \mathfrak{s u}_{n, 1}$-submodule. It should be treated as a module of the holomorphic discrete series for $U_{q} \mathfrak{s u}{ }_{n, 1}$.

Before we proceed further, let us fix more notation. The space $\mathbb{C}\left[\mathbb{C}^{n}\right]_{q}$ (as well as $\left.\mathcal{F}\left(U_{n}\right)_{q}\right)$ admits two different $U_{q} \mathfrak{s u}_{n, 1}$-actions, namely, the original one which was introduced earlier in Proposition 2.1] and the twisted one which appeared in this subsection. In order to emphasize that a space is considered together with the twisted $U_{q} \mathfrak{s u}_{n, 1}$-action $\pi_{\alpha}$ we shall add the subscript $\alpha$ in the notation, for example, $\mathbb{C}\left[\mathbb{C}^{n}\right]_{q, \alpha}$. The subscript will also indicate the pre-Hilbert space structure given by the scalar product $(\cdot, \cdot)_{\alpha, q}$.

The following important observation relates the usual and the twisted $U_{q} \mathfrak{s u}{ }_{n, 1}$-actions: the multiplication map $\mathcal{F}\left(U_{n}\right)_{q} \otimes \mathcal{F}\left(U_{n}\right)_{q, \alpha} \rightarrow \mathcal{F}\left(U_{n}\right)_{q, \alpha}$ is a morphism of $U_{q} \mathfrak{s u}{ }_{n, 1}$-modules. This can be proved by direct computations.

\subsection{Toeplitz operators}

In this subsection we produce $q$-analogues of Toeplitz operators with polynomial and finite symbols. As in the previous subsection, it is convenient to consider symbols from the algebra $\mathcal{F}\left(U_{n}\right)_{q}$ when there is no necessity to consider the cases of polynomial and finite symbols separately.

Let $P_{\alpha, q}$ be the orthogonal projection in $L^{2}\left(d m_{\alpha, q}\right)$ onto $L_{a}^{2}\left(d m_{\alpha, q}\right)$. The Toeplitz operator $T_{f}$ with the symbol $f \in \mathcal{F}\left(U_{n}\right)_{q}$ is defined as follows

$$
T_{f}: \mathbb{C}\left[\mathbb{C}^{n}\right]_{q, \alpha} \rightarrow \mathbb{C}\left[\mathbb{C}^{n}\right]_{q, \alpha}, \quad T_{f}(\psi)=P_{\alpha, q}(f \cdot \psi)
$$

To formulate the principal result of the present subsection, we recall the following well known construction. Let $A$ be a Hopf algebra and $V$ an $A$-module. Then the space $\operatorname{End}(V)$ admits the following 'canonical' structure of $A$-module: for $\xi \in A, T \in \operatorname{End}(V)$

$$
\xi(T)=\sum_{j} \xi_{j}^{\prime} \cdot T \cdot S\left(\xi_{j}^{\prime \prime}\right),
$$

where $S$ is the antipode of $A, \Delta(\xi)=\sum_{j} \xi_{j}^{\prime} \otimes \xi_{j}^{\prime \prime}$ (with $\Delta$ being the comultiplication), and the elements in the right-hand side are multiplied within the algebra $\operatorname{End}(V)$. This action of $A$ in $\operatorname{End}(V)$ makes $\operatorname{End}(V)$ into an $A$-module algebra.

Proposition 3.3 The linear map

$$
\mathcal{F}\left(U_{n}\right)_{q} \rightarrow \operatorname{End}\left(\mathbb{C}\left[\mathbb{C}^{n}\right]_{q, \alpha}\right), \quad f \mapsto T_{f}
$$

is a morphism of $U_{q} \mathfrak{s u}_{n, 1}$-modules.

Proof. In the classical case the projection $P_{\alpha, q}$ intertwines the twisted $\widetilde{S U(n, 1)}$-action in $L^{2}\left(d m_{\alpha}\right)$ and $L_{a}^{2}\left(d m_{\alpha}\right)$. In the quantum case the $U_{q} \mathfrak{s u}{ }_{n, 1}$-action $\pi_{\alpha}$ is not defined on 
the entire $L^{2}\left(d m_{\alpha, q}\right)$. Nevertheless, the intertwining property may be formulated due to the equality

$$
P_{\alpha, q}\left(\mathcal{F}\left(U_{n}\right)_{q, \alpha}\right)=\mathbb{C}\left[\mathbb{C}^{n}\right]_{q, \alpha} .
$$

To prove the equality, we endow the space $\mathbb{C}\left[\mathbb{C}^{n}\right]_{q, \alpha}$ with the obvious $\mathbb{Z}_{\geq 0}$-grading by powers of monomials and observe that each $f \in \mathcal{F}\left(U_{n}\right)_{q, \alpha}$ is orthogonal to all but finitely many homogeneous components of $\mathbb{C}\left[\mathbb{C}^{n}\right]_{q, \alpha}$. Now it is evident that the oper-

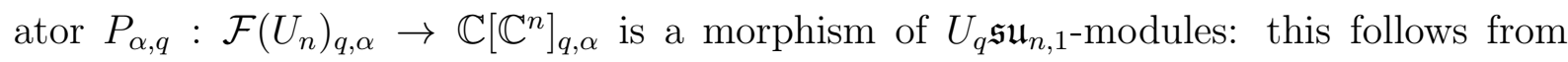
$U_{q} \mathfrak{s u}_{n, 1}$-invariance of the orthogonal complement of $\mathbb{C}\left[\mathbb{C}^{n}\right]_{q, \alpha}$ in $\mathcal{F}\left(U_{n}\right)_{q, \alpha}$.

Remark. One can use the above observation to show that the projection $P_{\alpha, q}$ is a $q$ integral operator with a simple kernel which is a $q$-analogue of the so-called Bergman kernel; see [10]. But this result is not needed in the present paper.

Let us get back to proving the proposition. Let $f \in \mathcal{F}\left(U_{n}\right)_{q}$. Denote by $\hat{f}=\hat{f}_{\alpha}$ the endomorphism of $\mathcal{F}\left(U_{n}\right)_{q, \alpha}$ given by $\hat{f}_{\alpha}(\varphi)=f \cdot \varphi$. The map

$$
\mathcal{F}\left(U_{n}\right)_{q} \rightarrow \operatorname{End}\left(\mathcal{F}\left(U_{n}\right)_{q, \alpha}\right), \quad f \mapsto \hat{f}_{\alpha}
$$

is a morphism $U_{q} \mathfrak{s} \mathfrak{u}_{n, 1}$-modules (see the remark at the end of the previous subsection). It remains to use the equality $T_{f}=\left.P_{q, \alpha} \cdot \hat{f}_{\alpha}\right|_{\mathbb{C}\left[\mathbb{C}^{n}\right]_{q, \alpha}}$.

\subsection{On the case of polynomial symbols}

For purposes of this paper we need mostly Toeplitz operators with finite symbols. However, we present some results about the polynomial case which seem to be interesting by themselves.

Let $\hat{z}_{i}$ stand for the operator in $\mathbb{C}\left[\mathbb{C}^{n}\right]_{q, \alpha}$ of the left multiplication by $z_{i}$, $\hat{z}_{i}^{*}$ for the adjoint operator. The following observation is straightforward: for a polynomial $f=$ $\sum a_{\underline{l}, \underline{m}} \mathbf{z}^{*} \mathbf{z}^{\underline{m}}$ one has $T_{f}=\sum a_{\underline{l}, \underline{\underline{\mathbf{z}}}} \hat{\mathbf{z}}^{*} \underline{\mathbf{z}} \hat{\mathbf{m}}^{\underline{m}}$. Thus all Toeplitz operators with polynomial symbols belong to the unital subalgebra in $\operatorname{End}\left(\mathbb{C}\left[\mathbb{C}^{n}\right]_{q, \alpha}\right)$ generated by $\hat{z}_{i}$ and $\hat{z}_{i}^{*}, i=$ $1,2, \ldots n$. Denote this subalgebra by $\mathcal{P}_{n, q, \alpha}$. It is an involutive algebra with the involution given by $*: \hat{z}_{i} \mapsto \hat{z}_{i}^{*}$.

Proposition 3.4 The $U_{q} \mathfrak{s u}_{n, 1}$-action in $\operatorname{End}\left(\mathbb{C}\left[\mathbb{C}^{n}\right]_{q, \alpha}\right)$ induces a $U_{q} \mathfrak{s u} \mathfrak{u}_{n, 1}$-module algebra structure in $\mathcal{P}_{n, q, \alpha}$.

Proof. First we have to establish $U_{q} \mathfrak{s u}_{n, 1}$-invariance of the subspace $\mathcal{P}_{n, q, \alpha}$ in $\operatorname{End}\left(\mathbb{C}\left[\mathbb{C}^{n}\right]_{q, \alpha}\right)$, that is, to show that $\xi\left(\hat{z}_{i}^{* a} \hat{z}_{j}^{b}\right)$ belong to $\mathcal{P}_{n, q, \alpha}$ for any $a, b \in \mathbb{Z}_{\geq 0}$, $i, j=1,2, \ldots n$, and $\xi \in U_{q} \mathfrak{s u}_{n, 1}$. According to proposition 3.3

$$
\xi\left(\hat{z}_{i}^{* a} \hat{z}_{j}^{b}\right)=\xi\left(T_{z_{i}^{* a} z_{j}^{b}}\right)=T_{\xi\left(z_{i}^{* a} z_{j}^{b}\right)}
$$

By the remark, preceding the present proposition, $T_{\xi\left(z_{i}^{* a} z_{j}^{b}\right)} \in \mathcal{P}_{n, q, \alpha}$. It remains to prove module algebra property (2.4) for $\mathcal{P}_{n, q, \alpha}$. But it may be derived easily from Proposition 3.2 
Proposition 3.5 The following commutation relations hold

$$
\begin{gathered}
\hat{z}_{i} \hat{z}_{j}=q \hat{z}_{j} \hat{z}_{i}, \quad i<j, \\
\hat{z}_{i}^{*} \hat{z}_{j}=q \hat{z}_{j} \hat{z}_{i}^{*}+q^{2 \alpha+2 n}\left(\hat{z}_{i}^{*} \hat{z}_{j}\left(q^{2}+\left(1-q^{2}\right) \sum_{k=1}^{n} \hat{z}_{k} \hat{z}_{k}^{*}\right)-q \hat{z}_{j} \hat{z}_{i}^{*}\right), \quad i \neq j, \\
\hat{z}_{j}^{*} \hat{z}_{j}=q^{2} \hat{z}_{j} \hat{z}_{j}^{*}+\left(1-q^{2}\right)\left(1-\sum_{k=j+1}^{n} \hat{z}_{k} \hat{z}_{k}^{*}\right) \\
+q^{2 \alpha+2 n}\left(\hat{z}_{j}^{*} \hat{z}_{j}\left(q^{2}+\left(1-q^{2}\right) \sum_{k=1}^{n} \hat{z}_{k} \hat{z}_{k}^{*}\right)-q^{2} \hat{z}_{j} \hat{z}_{j}^{*}-\left(1-q^{2}\right) \sum_{k=1}^{j} \hat{z}_{k} \hat{z}_{k}^{*}\right) .
\end{gathered}
$$

Proof. The relations may be deduced from the formulas

$$
\hat{z}_{i}\left(\mathbf{z}^{\underline{m}}\right)=q^{m_{n}+m_{n-1}+\ldots+m_{i+1}} \mathbf{z}^{\underline{m^{\prime}}}
$$

(where $\left.\underline{m^{\prime}}=\left(m_{1}, \ldots, m_{i}+1, \ldots, m_{n}\right)\right)$,

$$
\begin{gathered}
\hat{z}_{i}^{*}\left(\mathbf{z}^{\underline{m}}\right)=0, \quad m_{i}=0, \\
\hat{z}_{i}^{*}\left(\mathbf{z}^{\underline{m}}\right)=\frac{q^{m_{n}+m_{n-1}+\ldots+m_{i+1}}\left(1-q^{2 m_{i}}\right)}{1-q^{2 m_{1}+2 m_{2}+\ldots+2 m_{n}+2 n+2 \alpha}} \mathbf{z}^{\underline{\underline{m}^{\prime \prime}}}
\end{gathered}
$$

(where $\left.\underline{m^{\prime \prime}}=\left(m_{1}, \ldots, m_{i}-1, \ldots, m_{n}\right)\right)$. The first equality is obvious, the other follow from Proposition 3.1

Note that for $\alpha=\infty$ the above relations coincides with the defining relations for $\mathcal{P}\left(\mathbb{C}^{n}\right)_{q}$.

The unital involutive algebra given by the above commutation relations can be viewed as a two-parameter deformation of the polynomial algebra on $\mathbb{C}^{n}$. It is easy to show that for $n=1$ the algebra is isomorphic to the one considered in details in [6] (see the Introduction).

\subsection{Covariant symbols and $q$-Berezin transform}

In this subsection, we define the notion of covariant symbols of operators on the $q$-weighted Bergman spaces and use it to define a $q$-analogue of the Berezin transform.

To define covariant symbols we need certain inner product in the space of operators on a $q$-weighted Bergman space (see the Introduction). This product is defined via the so-called $q$-trace. So we recall first its definition.

Let $V$ be a finite-dimensional $U_{q} \mathfrak{s l}_{n+1}$-module. The $q$-trace is the linear functional on $\operatorname{End}(V)$ given by

$$
\operatorname{tr}_{q}: T \mapsto \operatorname{tr}\left(T \cdot \prod_{j=1}^{n} K_{j}^{-j(n+1-j)}\right) .
$$


The following well known observation explains the importance of this functional (see [17]): the $q$-trace is an invariant linear functional on $\operatorname{End}(V)$, i.e. $\operatorname{tr}_{q}(\xi(T))=\varepsilon(\xi) \cdot \operatorname{tr}_{q}(T)$ for any $\xi \in U_{q} \mathfrak{s l}_{n+1}$ and $T \in \operatorname{End}(V)$.

We will modify the definition slightly for the infinite-dimensional space $V=\mathbb{C}\left[\mathbb{C}^{n}\right]_{q, \alpha}$. Let $\operatorname{End}_{0}\left(\mathbb{C}\left[\mathbb{C}^{n}\right]_{q, \alpha}\right)$ be the subspace in $\operatorname{End}\left(\mathbb{C}\left[\mathbb{C}^{n}\right]_{q, \alpha}\right)$ of those automorphisms whose matrices in the basis $\left\{\mathbf{z}^{\underline{m}}\right\}$ have only finitely many non-zero entries. The $q$-trace (3.9) is a well defined linear functional on $\operatorname{End}_{0}\left(\mathbb{C}\left[\mathbb{C}^{n}\right]_{q, \alpha}\right)$. The invariance property still holds in this case.

Proposition 3.6 The q-trace is an invariant linear functional on $\operatorname{End}_{0}\left(\mathbb{C}\left[\mathbb{C}^{n}\right]_{q, \alpha}\right)$.

Sketch of a proof. A standard proof in the finite-dimensional case uses the canonical isomorphism of $U_{q} \mathfrak{s l}_{n+1}$-modules $\operatorname{End}(V) \simeq V \otimes V^{*}$ with $V^{*}$ being the dual $U_{q} \mathfrak{s l}_{n+1^{-}}$ module [17. The statement of Proposition 3.6 can be proved by the same argument since $\operatorname{End}_{0}\left(\mathbb{C}\left[\mathbb{C}^{n}\right]_{q, \alpha}\right) \simeq \mathbb{C}\left[\mathbb{C}^{n}\right]_{q, \alpha} \otimes \mathbb{C}\left[\mathbb{C}^{n}\right]_{q, \alpha}^{*}$.

Remarks. i) The formula (3.9) is the same as the one which defines the invariant integral on the quantum ball (see Proposition 2.4). But Proposition 2.4 does not follow formally from the above statement since the $\mathcal{D}\left(U_{n}\right)_{q}$-module $H$ is not a $U_{q} \mathfrak{s l}_{n+1}$-module.

ii) Using the equality (3.12) below and the same idea as in the proof of Proposition 2.5 one can show that the scalar product in $\operatorname{End}_{0}\left(\mathbb{C}\left[\mathbb{C}^{n}\right]_{q, \alpha}\right)$ given by $\left(T_{1}, T_{2}\right)=\operatorname{tr}_{q}\left(T_{2}^{*} \cdot T_{1}\right)$ is $U_{q} \mathfrak{s u} u_{n, 1}$-invariant. The unitary representation of $U_{q} \mathfrak{s u} u_{n, 1}$ in $\operatorname{End}_{0}\left(\mathbb{C}\left[\mathbb{C}^{n}\right]_{q, \alpha}\right)$ should be treated as a $q$-analogue of the canonical representation of $S U(n, 1)$ [23].

For a particular $\alpha$, we shall use the modified $q$-trace $\operatorname{Tr}_{q}$ on $\operatorname{End}_{0}\left(\mathbb{C}\left[\mathbb{C}^{n}\right]_{q, \alpha}\right)$ which differs from $\operatorname{tr}_{q}$ by a constant:

$$
\operatorname{Tr}_{q}=q^{n(n+\alpha+1)} \frac{\left(q^{2} ; q^{2}\right)_{n}}{\left(q^{2+2 \alpha} ; q^{2}\right)_{n}} \operatorname{tr}_{q}
$$

It is easy to deduce the following explicit formula for $\operatorname{Tr}_{q}$. Let $A \in \operatorname{End}_{0}\left(\mathbb{C}\left[\mathbb{C}^{n}\right]_{q, \alpha}\right)$ be the endomorphism given by $A\left(\mathbf{z}^{\underline{m}}\right)=\sum_{\underline{k}} A_{\underline{k}}^{\underline{m}} \cdot \mathbf{z}^{\underline{k}}$. Then

$$
\operatorname{Tr}_{q}(A)=\frac{\left(q^{2} ; q^{2}\right)_{n}}{\left(q^{2+2 \alpha} ; q^{2}\right)_{n}} \cdot \sum_{\underline{m}} A_{\underline{m}}^{\frac{m}{m}} \cdot q^{-2 n\left(m_{1}+m_{2}+\ldots+m_{n}\right)} \cdot q^{2 m_{2}+\ldots+2 m_{n}} \cdot \ldots \cdot q^{2 m_{n}}
$$

Now we are ready to define the notion of covariant symbols. Let $T \in \operatorname{End}\left(\mathbb{C}\left[\mathbb{C}^{n}\right]_{q, \alpha}\right)$. A distribution $\sigma(T) \in \mathcal{D}\left(U_{n}\right)_{q}^{\prime}$ is said to be the covariant symbol of $T$ if for any $f \in \mathcal{D}\left(U_{n}\right)_{q}$

$$
\int_{U_{n}} \sigma(T) \cdot f d \nu_{q}=\operatorname{Tr}_{q}\left(T \cdot T_{f}\right)
$$

Note that for $f \in \mathcal{D}\left(U_{n}\right)_{q}$ one has $T_{f} \in \operatorname{End}_{0}\left(\mathbb{C}\left[\mathbb{C}^{n}\right]_{q, \alpha}\right)$. Thus the right-hand side of (3.11) is well defined. The arguments cited at the end of subsection 2.4 imply existence 
and uniqueness of the covariant symbol of an arbitrary endomorphism $F$. Actually, the map $T \mapsto \sigma(T)$ is conjugated to the map $f \mapsto T_{f}$.

We have the following elementary property of the $q$-trace, which can be proved by the same arguments as the formula of integrating by parts (see the proof of Proposition 2.5): for any $T \in \operatorname{End}\left(\mathbb{C}\left[\mathbb{C}^{n}\right]_{q, \alpha}\right), T_{0} \in \operatorname{End}_{0}\left(\mathbb{C}\left[\mathbb{C}^{n}\right]_{q, \alpha}\right)$, and $\xi \in U_{q} \mathfrak{s u}_{n, 1}$

$$
\operatorname{Tr}_{q}\left(\xi(T) \cdot T_{0}\right)=\operatorname{Tr}_{q}\left(T \cdot S(\xi)\left(T_{0}\right)\right)
$$

The formula implies

Proposition 3.7 The map

$$
\operatorname{End}\left(\mathbb{C}\left[\mathbb{C}^{n}\right]_{q, \alpha}\right) \rightarrow \mathcal{D}\left(U_{n}\right)_{q}^{\prime}, \quad T \mapsto \sigma(T)
$$

is a morphism of $U_{q} \mathfrak{s u}_{n, 1}$-modules.

Proof. The equality (3.12) gives an identification of the $U_{q} \mathfrak{s u}_{n, 1}$-module $\operatorname{End}\left(\mathbb{C}\left[\mathbb{C}^{n}\right]_{q, \alpha}\right)$ with the dual of $\operatorname{End}_{0}\left(\mathbb{C}\left[\mathbb{C}^{n}\right]_{q, \alpha}\right.$ ) (see the definition of the dual module in subsection 2.4 ). What remains is to use Proposition 3.3 and the observation that the map $\operatorname{End}\left(\mathbb{C}\left[\mathbb{C}^{n}\right]_{q, \alpha}\right) \rightarrow$ $\mathcal{D}\left(U_{n}\right)_{q}^{\prime}, T \mapsto \sigma(T)$ is conjugated to $\mathcal{D}\left(U_{n}\right)_{q} \rightarrow \operatorname{End}_{0}\left(\mathbb{C}\left[\mathbb{C}^{n}\right]_{q, \alpha}\right), f \mapsto T_{f}$.

We are in position to define the $q$-Berezin transform $B_{q, \alpha}$. It is defined as the linear map from $\mathcal{D}\left(U_{n}\right)_{q}$ to $\mathcal{D}\left(U_{n}\right)_{q}^{\prime}$ which sends a finite function to the covariant symbol of the corresponding Toeplitz operator:

$$
B_{q, \alpha}: f \mapsto \sigma\left(T_{f}\right)
$$

The following crucial statement is straightforward.

Proposition 3.8 The $q$-Berezin transform is a morphism of $U_{q} \mathfrak{s u}_{n, 1}$-modules.

Due to Proposition 2.3, any morphism of $U_{q} \mathfrak{s u}_{n, 1}$-modules $T: \mathcal{D}\left(U_{n}\right)_{q} \rightarrow \mathcal{D}\left(U_{n}\right)_{q}^{\prime}$ is completely determined by the element $T\left(f_{0}\right) \in \mathcal{D}\left(U_{n}\right)_{q}^{\prime}$. Thus it would be very useful to compute $B_{q, \alpha}\left(f_{0}\right)$.

\section{Proposition 3.9}

$$
B_{q, \alpha}\left(f_{0}\right)=\left(q^{2 \alpha+2} ; q^{2}\right)_{n} \cdot y_{1}^{\alpha+n+1} .
$$

Proof. We have to check that for any $f \in \mathcal{D}\left(U_{n}\right)_{q}$

$$
\left(q^{2 \alpha+2} ; q^{2}\right)_{n} \int_{U_{n}} y_{1}^{\alpha+n+1} \cdot f d \nu_{q}=\operatorname{Tr}_{q}\left(T_{f_{0}} \cdot T_{f}\right)
$$


Let us denote by $f_{\underline{i}, \underline{k}}^{\underline{k}}\left(\underline{k} \in P(n), \underline{i}, \underline{j} \in \mathbb{Z}_{\geq 0}^{n}, \underline{i} \times \underline{j}=\underline{0}\right)$ the finite function given by

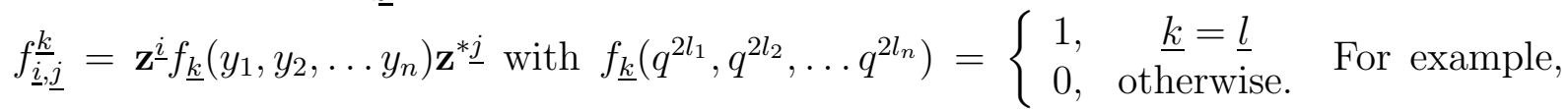
$f_{\underline{0}, \underline{0}}^{\underline{0}}=f_{0}$. Evidently, the functions $f_{\underline{i}, \underline{j}}^{\underline{k}}$ constitute a basis in $\mathcal{D}\left(U_{n}\right)_{q}$.

It follows from the definitions that the both hand sides of (3.13) vanish for $f:=f_{\underline{i}, \underline{k}}$ provided $\underline{i} \neq \underline{0}$ or $\underline{j} \neq \underline{0}$. Thus we have to verify (3.13) for $f:=f_{\underline{k}}$. It follows from (2.18) that

$$
\left(q^{2 \alpha+2} ; q^{2}\right)_{n} \int_{U_{n}} y_{1}^{\alpha+n+1} \cdot f_{\underline{k}} d \nu_{q}=\left(q^{2 \alpha+2} ; q^{2}\right)_{n} \cdot\left(q^{2} ; q^{2}\right)_{n} \cdot q^{2 k_{1}(\alpha+1)} \cdot q^{2 k_{2}} \cdot \ldots \cdot q^{2 k_{n}} .
$$

Let us compute the right-hand side of (3.13) with $f:=f_{\underline{k}}$. Evidently,

$$
f_{0} \cdot \mathbf{z}^{\underline{m}}=\left\{\begin{array}{cc}
f_{0}, & \underline{m}=\underline{0} \\
0, & \text { otherwise }
\end{array}\right.
$$

Besides, $\int_{U_{n}} f_{0} d m_{\alpha, q}=\left(q^{2 \alpha+2} ; q^{2}\right)_{n}$. These equalities mean that

$$
T_{f_{0}}\left(\mathbf{z}^{\underline{m}}\right)=\left\{\begin{array}{cc}
\left(q^{2 \alpha+2} ; q^{2}\right)_{n}, & \underline{m}=\underline{0} \\
0, & \text { otherwise. }
\end{array}\right.
$$

Clearly, the vector $f_{\underline{k}} \cdot \mathbf{z}^{\underline{m}} \in L^{2}\left(d m_{\alpha, q}\right)$ is orthogonal to any monomial $\mathbf{z}^{\underline{l}}$ except the case $\underline{l}=\underline{m}$. In particular, the monomials are eigenvectors of $T_{f_{\underline{k}}}$. Thus

$$
\begin{gathered}
\operatorname{Tr}_{q}\left(T_{f_{0}} \cdot T_{f_{\underline{k}}}\right)=\left(q^{2} ; q^{2}\right)_{n}\left(T_{f_{\underline{k}}}(1), 1\right)_{\alpha, q}=\left(q^{2} ; q^{2}\right)_{n} \int_{U_{n}} f_{\underline{k}} d m_{\alpha, q}= \\
=\left(q^{2 \alpha+2} ; q^{2}\right)_{n} \cdot\left(q^{2} ; q^{2}\right)_{n} \cdot q^{2 k_{1}(\alpha+1)} \cdot q^{2 k_{2}} \cdot \ldots \cdot q^{2 k_{n}} .
\end{gathered}
$$

\section{$4 q$-Laplace-Beltrami operator and associated q- spherical transform}

In this section we define a $q$-analogue of the $S U(n .1)$-invariant Laplace operator (the Laplace-Beltrami operator) on the unit ball and study a $q$-analogue of the spherical transform in the unit ball. Namely, we calculate the 'radial part' of the $q$-Laplace-Beltrami operator, find $q$-spherical functions, and present an inversion formula for the $q$-spherical transform.

\section{1 $q$-Laplace-Beltrami operator}

In this subsection, we consider the asymptotic expansion of the $q$-Berezin transform $B_{q, \alpha}$ at the limit $t=q^{2 \alpha} \rightarrow 0$. A $q$-analogue of the Laplace-Beltrami operator on the ball is defined as the coefficient at $t$ in that expansion. 
Recall (see the proof of Proposition [3.9) the notation $f_{\underline{i}, \underline{k}}^{\underline{k}}\left(\underline{k} \in P(n), \underline{i}, \underline{j} \in \mathbb{Z}_{\geq 0}^{n}\right.$, $\underline{i} \times \underline{j}=\underline{0})$. Suppose $T$ is the linear map from $\mathcal{D}\left(U_{n}\right)_{q}$ to $\mathcal{D}\left(U_{n}\right)_{q}^{\prime}$. The numbers

$$
T_{\underline{i}, \underline{\underline{k}}, \underline{\underline{p}}, \underline{\underline{s}} \underline{\underline{s}}}=\int_{U_{n}} T\left(f_{\underline{\underline{i}}, \underline{\underline{j}}}^{\underline{k}}\right) \cdot f_{\underline{\underline{p}}, \underline{\underline{r}}}^{\underline{s}} d \nu_{q} .
$$

will be called the matrix entries of $T$. Due to non-degeneracy of the pairing $\mathcal{D}\left(U_{n}\right)_{q}^{\prime} \times$ $\mathcal{D}\left(U_{n}\right)_{q} \rightarrow \mathbb{C}$ (subsection 2.4), the matrix entries determine $T$ completely.

Let us denote the matrix entries of the $q$-Berezin transform $B_{q, \alpha}$ by $B_{\underline{\underline{z}}, \underline{\underline{k}} \underline{\underline{j}}, \underline{\underline{p}}, \underline{\underline{s}}}(\alpha)$. Intro-

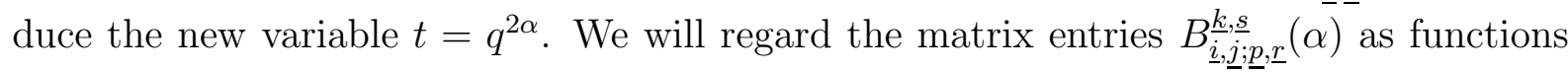

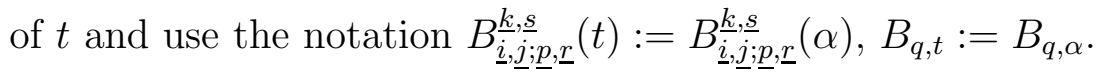

Proposition 4.1 There exists a sequence $\left\{B_{q, j}\right\}_{j \in \mathbb{N}}$ of linear endomorphisms of $\mathcal{D}\left(U_{n}\right)_{q}$ such that

i) $B_{q, j}$ are independent of $t$ (i.e. their matrix entries are independent of $t$ );

ii) each $B_{q, j}$ is a $U_{q} \mathfrak{s u}_{n, 1}$-module morphism;

iii) for any $f \in \mathcal{D}\left(U_{n}\right)_{q}$

$$
B_{q, t}(f)=f+\sum_{j=1}^{\infty} B_{q, j}(f) \cdot t^{j}
$$

where the series is convergent in $\mathcal{D}\left(U_{n}\right)_{q}^{\prime}$.

Proof. First of all, we will construct a sequence $\left\{B_{q, j}\right\}_{j \in \mathbb{Z} \geq 0}$ of linear operators from $\mathcal{D}\left(U_{n}\right)_{q}$ to $\mathcal{D}\left(U_{n}\right)_{q}^{\prime}$ such that each $B_{q, j}$ is independent of $t$ and $B_{q, t}(f)=\sum_{j=0}^{\infty} B_{q, j}(f) \cdot t^{j}$ for any $f \in \mathcal{D}\left(U_{n}\right)_{q}$ (the series is convergent in the sense that all the corresponding series of matrix entries are convergent). For that purpose we need

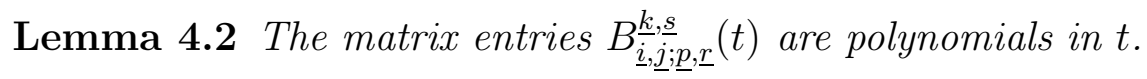

Proof of the lemma. Due to Proposition [2.3. for any $\underline{k}, \underline{i}, \underline{j} \in \mathbb{Z}_{\geq 0}^{n}(\underline{i} \times \underline{j}=\underline{0})$ there exists $\xi_{\underline{i}, \underline{j}}^{\underline{k}} \in U_{q} \mathfrak{s u}_{n, 1}$ such that $f_{\underline{i}, \underline{j}}^{k}=\xi_{\underline{i}, \underline{j}}^{\underline{k}}\left(f_{0}\right)$. Then,

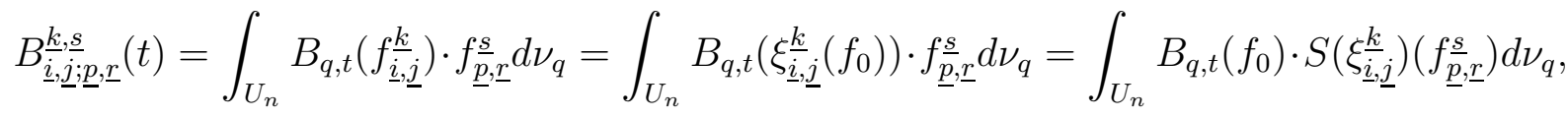

where the last equality is due to Proposition 3.8 and the equality (2.20). Thus to prove

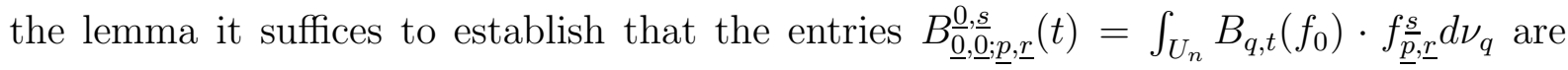
polynomials.

Recall (Proposition 3.9) that $B_{q, \alpha}\left(f_{0}\right)=\left(q^{2 \alpha+2} ; q^{2}\right)_{n} \cdot y_{1}^{\alpha+n+1}$. Thus

$$
\int_{U_{n}} B_{q, t}\left(f_{0}\right) \cdot f_{\underline{\underline{p}}, \underline{s}}^{\underline{s}} d \nu_{q}=\left(q^{2 \alpha+2} ; q^{2}\right)_{n} \int_{U_{n}} y_{1}^{\alpha+n+1} \cdot f_{\underline{\underline{p}}, \underline{\underline{s}}}^{\underline{\underline{s}}} d \nu_{q} .
$$


The latter integral vanishes with $\underline{p} \neq \underline{0}$ or $\underline{r} \neq \underline{0}$. Consider the integral $\left(q^{2 \alpha+2} ; q^{2}\right)_{n} \int_{U_{n}} y_{1}^{\alpha+n+1} \cdot f_{\underline{k}} d \nu_{q}\left(f_{\underline{k}}\right.$ are defined in the proof of Proposition [3.9) $)$. Obviously,

$$
\begin{aligned}
\left(q^{2 \alpha+2} ; q^{2}\right)_{n} \int_{U_{n}} y_{1}^{\alpha+n+1} \cdot f_{\underline{k}} d \nu_{q} & =\left(q^{2 \alpha+2} ; q^{2}\right)_{n}\left(q^{2} ; q^{2}\right)_{n} q^{2 k_{1}(\alpha+1)} q^{2 k_{2}} \ldots q^{2 k_{n}}= \\
& =\left(q^{2} t ; q^{2}\right)_{n}\left(q^{2} ; q^{2}\right)_{n} q^{2 k_{1}} t^{k_{1}} q^{2 k_{2}} \ldots q^{2 k_{n}}
\end{aligned}
$$

Now we can define $\left\{B_{q, m}\right\}_{m \in \mathbb{Z}_{\geq 0}}$ by their matrix coefficients

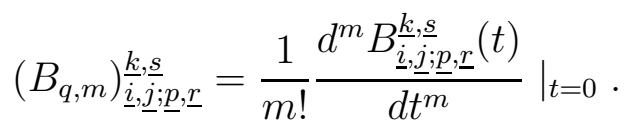

This definition implies the $U_{q} \mathfrak{s u} \mathfrak{s}_{n, 1}$-invariance of the maps $B_{q, m}$. Indeed, we should prove coincidence of the maps $B_{q, m} \cdot \xi$ and $\xi \cdot B_{q, m}$ for any $\xi \in U_{q} \mathfrak{s u}_{n, 1}$, or, equivalently, equality

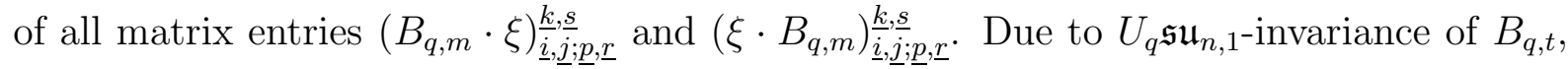
one has

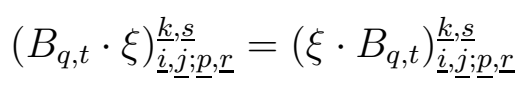

for any $\underline{i}, \underline{j}, \underline{p}, \underline{r}, \underline{k}, \underline{s}$. What remains is to differentiate the latter equality.

Let us show that the image of the map $B_{q, m}: \mathcal{D}\left(U_{n}\right)_{q} \rightarrow \mathcal{D}\left(U_{n}\right)_{q}^{\prime}$ is contained in $\mathcal{D}\left(U_{n}\right)_{q}$. Since all $B_{q, m}$ are morphisms of $U_{q} \mathfrak{s u}_{n, 1}$-modules, we need to verify that $B_{q, m}\left(f_{0}\right) \in \mathcal{D}\left(U_{n}\right)_{q}$. This can be easily deduced from the following equivalent formulation of Proposition 3.9 .

$$
B_{q, t}\left(f_{0}\right)=\left(q^{2} t ; q^{2}\right)_{n} \cdot \sum_{k=0}^{\infty} f_{k} q^{2 k(n+1)} t^{k}
$$

where $f_{k}, k \in \mathbb{Z}_{\geq 0}$, are the finite functions given by

$$
f_{k}=f_{k}\left(y_{1}, y_{2}, \ldots, y_{n}\right)=\left\{\begin{array}{lc}
1, & y_{1}=q^{2 k} \\
0, & \text { otherwise }
\end{array}\right.
$$

and the series (4.2) is convergent in $\mathcal{D}\left(U_{n}\right)_{q}^{\prime}$. Differentiating with respect to $t$ proves $B_{q, m}\left(f_{0}\right) \in \mathcal{D}\left(U_{n}\right)_{q}$.

The equality (4.2) and continuity of the $U_{q} \mathfrak{s u}$,-action in $\mathcal{D}\left(U_{n}\right)_{q}^{\prime}$ prove also statement iii) of Proposition 4.1.

Proposition 4.1 implies, in particular, that $B_{q, t}=\mathrm{id}+o(1)$ when $t \rightarrow 0$. By analogy with the classical case we call the first term of the asymptotic series (4.1) the $q$-LaplaceBeltrami operator on the quantum ball:

$$
\Delta_{n, q}=\left.\frac{q^{-2 n}}{1-q^{2}} \frac{d B_{q, t}}{d t}\right|_{t=0} .
$$

Clearly, $\Delta_{n, q}: \mathcal{D}\left(U_{n}\right)_{q} \rightarrow \mathcal{D}\left(U_{n}\right)_{q}$ is a morphism of $U_{q} \mathfrak{s u}{ }_{n, 1}$-modules.

There are many evidences that this operator should indeed be treated as a $q$-analogue of the classical Laplace-Beltrami operator in the unit ball. The results of the present provide an example of such an evidence. 
In conclusion, we make also the following remark. The operator $\Delta_{n, q}$ appeared as the first term of asymptotic of the $q$-Berezin transform. It turns out that other terms can be explicitly expressed via $\Delta_{n, q}$. This will be shown in the next section (subsection 5.2).

\subsection{Radial part of the $q$-Laplace-Beltrami operator}

In the classical case the Laplace-Beltrami operator in the unit ball keeps invariant the space of smooth radial functions, i. e. functions depending on the radius only. The reason is that the radial functions are precisely the $S(U(n) \times U(1))$-invariant functions with $S(U(n) \times U(1)) \subset S U(n, 1)$ being the isotropy group of the centre of the ball. Thus the 'right' $q$-analogue of the radial functions are functions on the quantum ball which are $U_{q} \mathfrak{s}\left(\mathfrak{u}_{n} \times \mathfrak{u}_{1}\right)$-invariant where $U_{q} \mathfrak{s}\left(\mathfrak{u}_{n} \times \mathfrak{u}_{1}\right)$ is the $*$-Hopf subalgebra in $U_{q} \mathfrak{s} \mathfrak{u}_{n, 1}$ generated by $E_{i}, F_{i}, i=1,2, \ldots, n-1$, and all $K_{j}^{ \pm 1}$ 's.

It can be proved that any $U_{q} \mathfrak{s}\left(\mathfrak{u}_{n} \times \mathfrak{u}_{1}\right)$-invariant element in $\mathcal{P}\left(\mathbb{C}^{n}\right)_{q}$ is a polynomial in $z_{1} z_{1}^{*}+z_{2} z_{2}^{*}+\ldots+z_{n} z_{n}^{*}$. The idea of the proof is as follows. $U_{q} \mathfrak{s}\left(\mathfrak{u}_{n} \times \mathfrak{u}_{1}\right)$-invariance of a polynomial, in particular, implies its $U_{q} \mathfrak{h}$-invariance, i. e. $K_{j}$-invariance for any $j$. Obviously, the latter means that the polynomial depends on $z_{1} z_{1}^{*}, z_{2} z_{2}^{*}, \ldots, z_{n} z_{n}^{*}$ only. One can write down without difficulties explicit formulas for the action of the generators $E_{i}, F_{i}, i=1,2, \ldots, n-1$, on an arbitrary element which depends on $z_{1} z_{1}^{*}, z_{2} z_{2}^{*}, \ldots, z_{n} z_{n}^{*}$ and find the invariant elements.

In many computations it is convenient to use the element $y_{1}=1-z_{1} z_{1}^{*}-\ldots-z_{n} z_{n}^{*}$ instead of $z_{1} z_{1}^{*}+z_{2} z_{2}^{*}+\ldots+z_{n} z_{n}^{*}$ since the former quasicommutes with all the generators $z_{i}, z_{i}^{*}($ see $(2.16))$ :

$$
z_{i} y_{1}=q^{-2} y_{1} z_{i}, \quad z_{i}^{*} y_{1}=q^{2} y_{1} z_{i}^{*}
$$

In the sequel we omit the subscript 1 in the notation for $y_{1}$.

Using precisely the same arguments, one can show that any $U_{q} \mathfrak{s}\left(\mathfrak{u}_{n} \times \mathfrak{u}_{1}\right)$-invariant finite function or distribution on the quantum ball depends on $y$ only.

Recall the notation $f_{k}, k \in \mathbb{Z}_{\geq 0}$, from the previous subsection:

$$
f_{k}(y)=\left\{\begin{array}{l}
1, \quad y=q^{2 k} \\
0, \quad \text { otherwise }
\end{array}\right.
$$

These functions constitute a basis in the space of radial finite functions on the quantum ball. They have the following obvious properties: first, $f_{k} \cdot f_{l}=\delta_{k l} f_{k}$ with $\delta_{k l}$ being the Kronecker symbol, second, for any distribution $f(y)$

$$
f=\sum_{k=0}^{\infty} f\left(q^{2 k}\right) \cdot f_{k}
$$

where the series converges in $\mathcal{D}\left(U_{n}\right)_{q}^{\prime}$.

Our aim now is to compute the action of the $q$-Laplace-Beltrami operator $\Delta_{n, q}$ on radial finite functions. We would determine the action completely if we find $\Delta_{n, q}\left(f_{k}\right)$. 
Proposition 4.3 The operator $\Delta_{n, q}$ has the following Jacobi matrix form

$$
\Delta_{n, q}\left(f_{k}\right)=\frac{q^{2}}{\left(1-q^{2}\right)^{2}} \cdot\left(\left(1-q^{2 k+2}\right) f_{k+1}-\left(1+q^{-2 n}-2 q^{2 k}\right) f_{k}+\left(q^{-2 n}-q^{2 k-2}\right) f_{k-1}\right)
$$

(we assume $f_{k} \equiv 0$ for $k<0$ ).

The proof of the proposition will be given in the subsection 4.4

Proposition 4.3 allows us to apply the $q$-Laplace-Beltrami operator to any radial function $f=f(y)$. We call restriction of the operator to the space of radial functions the radial part of the $q$-Laplace-Beltrami operator and denote it by $\Delta_{n, q}^{(\mathrm{r})}$. Using the explicit formula from Proposition 4.3. one can show that the radial part is given by the following second-order difference operator

$$
\Delta_{n, q}^{(\mathrm{r})}=\frac{q^{-n} y^{n+1}}{\left(y q^{2} ; q^{2}\right)_{n-1}} D y^{-n+1}\left(y q ; q^{2}\right)_{n} D
$$

with $D f(y)=\frac{f\left(q^{-1} y\right)-f(q y)}{q^{-1} y-q y}$.

\section{$4.3 \quad q$-Spherical transform}

In this subsection we describe eigenfunctions of the operator $\Delta_{n, q}^{(\mathrm{r})}$ and present an explicit formula for expansion in these functions. The associated $q$-spherical transform should be viewed as a $q$-analogue of the spherical transform in the unit ball [12]. The eigenfunctions of $\Delta_{n, q}^{(\mathrm{r})}$ appear to be closely related to certain one-parameter family of the Al-SalamChihara polynomials [13, and this observation simplifies proofs of many statements.

Recall the definition of the basic hypergeometric series ${ }_{3} \phi_{2}$ [20]:

$$
{ }_{3} \phi_{2}\left(\begin{array}{c}
a_{1}, \quad a_{2}, \quad a_{3} \\
b_{1}, \quad b_{2}
\end{array} \quad q, \quad z\right)=\sum_{n=0}^{\infty} \frac{\left(a_{1} ; q\right)_{n} \cdot\left(a_{2} ; q\right)_{n} \cdot\left(a_{3} ; q\right)_{n}}{\left(b_{1} ; q\right)_{n} \cdot\left(b_{2} ; q\right)_{n} \cdot(q ; q)_{n}} z^{n} .
$$

Recall also the notation $h=\log q^{-2}$ (see Introduction). We define the element $\phi_{\rho}(y) \in$ $\mathcal{D}\left(U_{n}\right)_{q}^{\prime}$ as follows

$$
\phi_{\rho}(y)={ }_{3} \phi_{2}\left(\begin{array}{cccc}
y^{-1}, & q^{n+i \rho}, & q^{n-i \rho} & q^{2}, \\
& q^{2 n}, & 0
\end{array}\right), \quad \rho \in\left[0 ; \frac{2 \pi}{h}\right] .
$$

Obviously, $\phi_{\rho}(1)=1$. It is a $q$-analogue of the spherical function in the unit ball as one can see from the following

Proposition 4.4 The distribution $\phi_{\rho}(y)$ is an eigenvector of the operator $\Delta_{n, q}^{(\mathrm{r})}$ :

$$
\Delta_{n, q}^{(\mathrm{r})}\left(\phi_{\rho}(y)\right)=\lambda(\rho) \cdot \phi_{\rho}(y)
$$

with $\lambda(\rho)=-q^{2-2 n} \frac{\left(1-q^{n+i \rho}\right)\left(1-q^{n-i \rho}\right)}{\left(1-q^{2}\right)^{2}}, \rho \in\left[0 ; \frac{2 \pi}{h}\right]$. 
Proof. Recall the notation $Q_{m}(x ; a, b \mid q)$ for the Al-Salam-Chihara polynomials [13, Section 3.8]. It is straightforward that

$$
\phi_{\rho}\left(q^{2 m}\right)=\frac{q^{n m}}{\left(q^{2 n} ; q^{2}\right)_{m}} \cdot Q_{m}\left(\cos \frac{h \rho}{2} ; q^{n}, q^{n} \mid q^{2}\right)
$$

The statement of the Proposition 4.4 is just another formulation of the recurrence relation for the Al-Salam-Chihara polynomials [13, (3.8.4)].

Let us compute restriction of the invariant integral in the quantum ball onto the space of radial finite functions.

$$
\begin{gathered}
\int_{U_{n}} f(y) d \nu_{q}=\left(q^{2} ; q^{2}\right)_{n} \cdot \sum_{\underline{k} \in P(n)} f\left(q^{2 k_{1}}\right) \cdot q^{-2 n k_{1}} \cdot q^{2 k_{2}} \cdot \ldots \cdot q^{2 k_{n}}= \\
=\left(q^{2} ; q^{2}\right)_{n} \cdot \sum_{k=0}^{\infty} f\left(q^{2 k}\right) \cdot q^{-2 n k} \sum_{0 \leq k_{n} \leq \ldots \leq k_{2} \leq k} q^{2 k_{2}} \cdot \ldots \cdot q^{2 k_{n}} .
\end{gathered}
$$

Using the formula (4.15) and the Jackson integral (3.5), we finally get

$$
\int_{U_{n}} f(y) d \nu_{q}=\frac{1-q^{2 n}}{1-q^{2}} \int_{0}^{1} f(y) y^{-n-1}\left(y q^{2} ; q^{2}\right)_{n-1} d_{q^{2}} y .
$$

Let us denote by $\mathcal{L}_{n, q}$ and $\mathcal{L}_{n, q}^{\prime}$ the spaces of radial finite functions and radial distributions on the quantum ball, respectively. Elements of these spaces can be treated as functions on the geometric progression $q^{2 \mathbb{Z}_{\geq 0}}$. We also impose the notation $\mathcal{L}_{n, q}^{2}$ for the Hilbert space of 'square integrable' radial distributions:

$$
\mathcal{L}_{n, q}^{2}=\left\{\left.f(y) \in \mathcal{L}_{n, q}^{\prime}\left|\|f\|_{\mathcal{L}^{2}}^{2}=\frac{1-q^{2 n}}{1-q^{2}} \int_{0}^{1}\right| f(y)\right|^{2} y^{-n-1}\left(y q^{2} ; q^{2}\right)_{n-1} d_{q^{2}} y<\infty\right\} .
$$

We define the $q$-spherical transform as the map $\mathcal{F}: \mathcal{L}_{n, q} \rightarrow C^{\infty}\left(0 ; \frac{2 \pi}{h}\right)$ given by

$$
f(y) \mapsto \mathcal{F} f(\rho)=\frac{1-q^{2 n}}{1-q^{2}} \int_{0}^{1} f(y) \phi_{\rho}(y) y^{-n-1}\left(y q^{2} ; q^{2}\right)_{n-1} d_{q^{2}} y .
$$

It is clear (see the proof of Proposition 4.4) that

$$
\begin{aligned}
& \mathcal{F} f_{k}(\rho)=\left(1-q^{2 n}\right) \cdot q^{-2 k n} \cdot\left(q^{2 k+2} ; q^{2}\right)_{n-1} \cdot \phi_{\rho}\left(q^{2 k}\right)= \\
= & \left(1-q^{2 n}\right) \cdot q^{-k n} \cdot \frac{\left(q^{2 k+2} ; q^{2}\right)_{n-1}}{\left(q^{2 n} ; q^{2}\right)_{k}} \cdot Q_{k}\left(\cos \frac{h \rho}{2} ; q^{n}, q^{n} \mid q^{2}\right) .
\end{aligned}
$$

Hence the image of $\mathcal{F}$ is the space of polynomials in $\cos \frac{h \rho}{2}$.

The following proposition is can be derived from the spectral decomposition of the operator $\Delta_{n, q}^{(\mathrm{r})}$, and a special case of a general result of [11, Section 5]. 
Proposition 4.5 i) The operator $\Delta_{n, q}^{(\mathrm{r})}$ on $\mathcal{L}_{n, q}$ can be extended to a bounded self-adjoint operator on $\mathcal{L}_{n, q}^{2}$. It has simple purely continuous spectrum which coincides with the segment $\left[\lambda\left(\frac{2 \pi}{h}\right) ; \lambda(0)\right]$ (with $\lambda$ being defined in Proposition 4.4).

ii) For any finite function $f(y)$

$$
f(y)=\frac{1}{4 \pi} \cdot \frac{h}{1-q^{2 n}} \cdot \int_{0}^{\frac{2 \pi}{h}} \mathcal{F} f(\rho) \phi_{\rho}(y) \frac{d \rho}{|c(\rho)|^{2}}
$$

where $c(\rho)$ (a q-analogue of the Harish-Chandra function) is given by

$$
c(\rho)=\frac{\Gamma_{q^{2}}(n) \Gamma_{q^{2}}(i \rho)}{\Gamma_{q^{2}}^{2}\left(\frac{n}{2}+\frac{i \rho}{2}\right)} .
$$

iii) The $q$-spherical transform $\mathcal{F}: \mathcal{L}_{n, q} \rightarrow C^{\infty}\left(0 ; \frac{2 \pi}{h}\right)$ can be extended to a unitary linear operator $\mathcal{F}: \mathcal{L}_{n, q}^{2} \rightarrow L^{2}\left(\frac{d \rho}{|c(\rho)|^{2}}\right):$

$$
\frac{1-q^{2 n}}{1-q^{2}} \int_{0}^{1}|f(y)|^{2} y^{-n-1}\left(y q^{2} ; q^{2}\right)_{n-1} d_{q^{2}} y=\frac{1}{4 \pi} \cdot \frac{h}{1-q^{2 n}} \cdot \int_{0}^{\frac{2 \pi}{h}}|\mathcal{F} f(\rho)|^{2} \frac{d \rho}{|c(\rho)|^{2}}
$$

(the Plancherel formula).

Note that, due to (4.10), statement iii) can be rewritten as the orthogonality relations for the Al-Salam-Chihara polynomials [13, Section 3.8]:

$$
\frac{1}{4 \pi} \int_{0}^{\frac{2 \pi}{h}} Q_{k}\left(\cos \frac{h \rho}{2} ; q^{n}, q^{n} \mid q^{2}\right) Q_{m}\left(\cos \frac{h \rho}{2} ; q^{n}, q^{n} \mid q^{2}\right) \frac{d \rho}{|c(\rho)|^{2}}=\delta_{k m} \cdot \frac{\left(q^{2 n} ; q^{2}\right)_{k}^{2}}{h\left(q^{2 k+2} ; q^{2}\right)_{n-1}} .
$$

\subsection{Proof of Proposition 4.3}

It suffices, due to (4.4), to verify that

$$
B_{q, t}\left(f_{k}\right)=f_{k}+t \frac{q^{2 n+2}}{\left(1-q^{2}\right)} \cdot\left(\left(1-q^{2 k+2}\right) f_{k+1}-\left(1+q^{-2 n}-2 q^{2 k}\right) f_{k}+\left(q^{-2 n}-q^{2 k-2}\right) f_{k-1}\right)
$$

modulo $t^{2}$. For that purpose we compute first $T_{f_{k}}$.

Recall the notation $\mathbb{C}\left[\mathbb{C}^{n}\right]_{q, \alpha}$ (subsection 3.1). Define a $\mathbb{Z}_{\geq 0}$-grading in the space $\mathbb{C}\left[\mathbb{C}^{n}\right]_{q, \alpha}$ as follows

$$
\mathbb{C}\left[\mathbb{C}^{n}\right]_{q, \alpha}^{(m)}=\text { linear span of } \mathbf{z}^{\underline{m}},|\underline{m}|=m \text {. }
$$

We need first 


\section{Lemma 4.6}

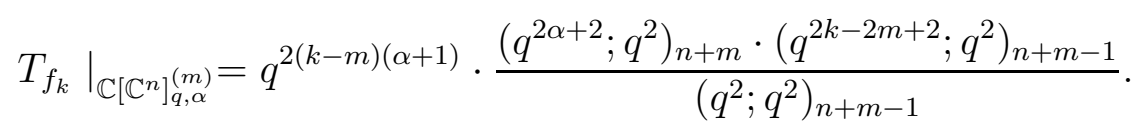

Proof of the lemma. Using the same arguments as in the classical case, it can be proved that $\mathbb{C}\left[\mathbb{C}^{n}\right]_{q, \alpha}^{(m)}, m \in \mathbb{Z}_{\geq 0}$, are pairwise non-isomorphic irreducible $U_{q} \mathfrak{s}\left(\mathfrak{u}_{n} \times \mathfrak{u}_{1}\right)$-modules (we mean restriction of the representation $\pi_{\alpha}$ onto $U_{q} \mathfrak{s}\left(\mathfrak{u}_{n} \times \mathfrak{u}_{1}\right)$ ). Due to the $U_{q} \mathfrak{s}\left(\mathfrak{u}_{n} \times \mathfrak{u}_{1}\right)$ invariance of $f_{k}$ (with respect to the untwisted $U_{q} \mathfrak{s}\left(\mathfrak{u}_{n} \times \mathfrak{u}_{1}\right)$-action), Proposition 3.3, and the Schur lemma ${ }^{3}$

$$
\left.T_{f_{k}}\right|_{\mathbb{C}\left[\mathbb{C}^{n}\right]_{q, \alpha}^{(m)}}=c_{k}^{m}
$$

To compute the constant $c_{k}^{m}$ we apply $T_{f_{k}}$ to a distinguished vector in $\mathbb{C}\left[\mathbb{C}^{n}\right]_{q, \alpha}^{(m)}$, for example, $z_{n}^{m}$ :

$$
c_{k}^{m}=\frac{\left(T_{f_{k}} z_{n}^{m}, z_{n}^{m}\right)_{\alpha, q}}{\left(z_{n}^{m}, z_{n}^{m}\right)_{\alpha, q}}=\frac{\left(f_{k} z_{n}^{m}, z_{n}^{m}\right)_{\alpha, q}}{\left(z_{n}^{m}, z_{n}^{m}\right)_{\alpha, q}} .
$$

By Proposition 3.1 the denominator is equal to $\frac{\left(q^{2} ; q^{2}\right)_{m}}{\left(q^{2 n+2 \alpha+2} ; q^{2}\right)_{m}}$. What remains is to compute the numerator:

$$
\begin{aligned}
& \left(T_{f_{k}} z_{n}^{m}, z_{n}^{m}\right)_{\alpha, q}=\int_{U_{n}} z_{n}^{* m} f_{k} z_{n}^{m} d m_{\alpha, q}=\int_{U_{n}} f_{k-m}\left(q^{2} y_{n} ; q^{2}\right)_{m} d m_{\alpha, q}= \\
& =\left(q^{2 \alpha+2} ; q^{2}\right)_{n} \sum_{\underline{k} \in P(n)} f_{k-m}\left(q^{2 k_{1}}\right)\left(q^{2+2 k_{n}} ; q^{2}\right)_{m} q^{2 k_{1}(\alpha+1)} q^{2 k_{2}} \ldots q^{2 k_{n}}= \\
& =\left(q^{2 \alpha+2} ; q^{2}\right)_{n} q^{2(k-m)(\alpha+1)} \sum_{0 \leq k_{n} \ldots \leq k_{2} \leq k-m}\left(q^{2+2 k_{n}} ; q^{2}\right)_{m} q^{2 k_{2}} \ldots q^{2 k_{n}}
\end{aligned}
$$

To continue computation we need the following simple formula which can be proved by induction:

$$
\sum_{a \leq l_{n} \ldots \leq l_{1} \leq b} q^{2 l_{1}} \ldots q^{2 l_{n}}=q^{2 a n} \frac{\left(q^{2 b-2 a+2} ; q^{2}\right)_{n}}{\left(q^{2} ; q^{2}\right)_{n}} .
$$

By this formula $\left(q^{2+2 k_{n}} ; q^{2}\right)_{m}=\left(q^{2} ; q^{2}\right)_{m} \sum_{0 \leq l_{m} \ldots \leq l_{1} \leq k_{n}} q^{2 l_{1}} \ldots q^{2 l_{m}}$. Applying (4.15) one more time, we finally get

$$
\left(T_{f_{k}} z_{n}^{m}, z_{n}^{m}\right)_{\alpha, q}=\left(q^{2 \alpha+2} ; q^{2}\right)_{n} q^{2(k-m)(\alpha+1)}\left(q^{2} ; q^{2}\right)_{m} \frac{\left(q^{2 k-2 m+2} ; q^{2}\right)_{n+m-1}}{\left(q^{2} ; q^{2}\right)_{n+m-1}} .
$$

\footnotetext{
${ }^{3}$ Since $\mathbb{C}\left[\mathbb{C}^{n}\right]_{q, \alpha}^{(m)}$ is an irreducible $U_{q} \mathfrak{s}\left(\mathfrak{u}_{n} \times \mathfrak{u}_{1}\right)$-module, the algebra homomorphism $U_{q} \mathfrak{s}\left(\mathfrak{u}_{n} \times \mathfrak{u}_{1}\right) \rightarrow$ $\operatorname{End}\left(\mathbb{C}\left[\mathbb{C}^{n}\right]_{q, \alpha}^{(m)}\right)$ is surjective, however the only operators commuting with the full matrix algebra are constants.
} 
Let $P_{m}$ denotes the orthogonal projection in $\mathbb{C}\left[\mathbb{C}^{n}\right]_{q, \alpha}$ onto $\mathbb{C}\left[\mathbb{C}^{n}\right]_{q, \alpha}^{(m)}$. The above lemma says that

$$
T_{f_{k}}=\sum_{m=0}^{k} q^{2(k-m)(\alpha+1)} \cdot \frac{\left(q^{2 \alpha+2} ; q^{2}\right)_{n+m} \cdot\left(q^{2 k-2 m+2} ; q^{2}\right)_{n+m-1}}{\left(q^{2} ; q^{2}\right)_{n+m-1}} \cdot P_{m}
$$

Lemma 4.7 The covariant symbol $\sigma\left(P_{m}\right)$ is given by

$$
\sigma\left(P_{m}\right)=q^{-2 m(\alpha+n+1)} \cdot \frac{\left(q^{2 \alpha+2 n+2} ; q^{2}\right)_{m}}{\left(q^{2} ; q^{2}\right)_{m}} \cdot y^{\alpha+n+1} \cdot\left(y q^{-2 m+2} ; q^{2}\right)_{m}
$$

Proof of the lemma. Since $\mathbb{C}\left[\mathbb{C}^{n}\right]_{q, \alpha}^{(m)}, m \in \mathbb{Z}_{\geq 0}$, are pairwise non-isomorphic irreducible $U_{q} \mathfrak{s}\left(\mathfrak{u}_{n} \times \mathfrak{u}_{1}\right)$-modules, the projections $P_{m}$ are $U_{q} \mathfrak{s}\left(\mathfrak{u}_{n} \times \mathfrak{u}_{1}\right)$-module morphisms. Thus by Proposition 3.7 $\sigma\left(P_{m}\right)$ should be a function of $y$. Denote it by $p_{m}(y)$. Recall that $p_{m}(y)=\sum_{k=0}^{\infty} p_{m}\left(q^{2 k}\right) f_{k}(y)$. The coefficients $p_{m}\left(q^{2 k}\right)$ can be derived from the equalities

$$
\int_{U_{n}} p_{m}(y) \cdot f_{l} d \nu_{q}=\operatorname{Tr}_{q}\left(P_{m} \cdot T_{f_{l}}\right), \quad l \in \mathbb{Z}_{\geq 0}
$$

Using the property $f_{k} \cdot f_{l}=\delta_{k l} f_{k}$ and lemma 4.6. we can rewrite the latter equality as follows:

$$
\begin{gathered}
p_{m}\left(q^{2 l}\right) \cdot\left(q^{2} ; q^{2}\right)_{n} \cdot q^{-2 n l} \cdot \sum_{0 \leq k_{n} \ldots \leq k_{2} \leq l} q^{2 k_{2}} \ldots q^{2 k_{n}}= \\
=q^{2(l-m)(\alpha+1)} \cdot \frac{\left(q^{2 \alpha+2} ; q^{2}\right)_{n+m} \cdot\left(q^{2 l-2 m+2} ; q^{2}\right)_{n+m-1}}{\left(q^{2} ; q^{2}\right)_{n+m-1}} \cdot \operatorname{Tr}_{q}\left(P_{m}\right) .
\end{gathered}
$$

By (3.10) $\operatorname{Tr}_{q}\left(P_{m}\right)=\frac{\left(q^{2} ; q^{2}\right)_{n}}{\left(q^{2 \alpha+2} ; q^{2}\right)_{n}} \cdot q^{-2 n m} \cdot \sum_{0 \leq k_{n} \ldots \leq k_{2} \leq m} q^{2 k_{2}} \ldots q^{2 k_{n}}$. Using (4.15) we obtain $p_{m}\left(q^{2 l}\right)=\frac{\left(q^{2 \alpha+2 n+2} ; q^{2}\right)_{m} \cdot\left(q^{2 l-2 m+2} ; q^{2}\right)_{m}}{\left(q^{2} ; q^{2}\right)_{m}} \cdot q^{2(l-m)(\alpha+n+1)}$.

We continue the proof of our proposition. Lemmas 4.6 and 4.7 now imply

$$
\begin{aligned}
B_{q, \alpha}\left(f_{k}\right)= & q^{2 k(\alpha+1)} \cdot y^{\alpha+n+1} \cdot \sum_{m=0}^{k} q^{-4 m(\alpha+1)-2 m n} . \\
& \cdot \frac{\left(q^{2 \alpha+2} ; q^{2}\right)_{n+m}\left(q^{2 k-2 m+2} ; q^{2}\right)_{n+m-1}\left(q^{2 \alpha+2 n+2} ; q^{2}\right)_{m}}{\left(q^{2} ; q^{2}\right)_{n+m-1}\left(q^{2} ; q^{2}\right)_{m}} \cdot\left(y q^{-2 m+2} ; q^{2}\right)_{m}
\end{aligned}
$$

Note that $\left(y q^{-2 m+2} ; q^{2}\right)_{m}=\sum_{l=0}^{\infty}\left(q^{2 l-2 m+2} ; q^{2}\right)_{m} \cdot f_{l}=\sum_{l=m}^{\infty}\left(q^{2 l-2 m+2} ; q^{2}\right)_{m} \cdot f_{l}$. Consequently

$$
\begin{aligned}
& y^{\alpha+n+1} \cdot\left(y q^{-2 m+2} ; q^{2}\right)_{m}=\sum_{l=m}^{\infty} q^{2 l(\alpha+n+1)} \cdot\left(q^{2 l-2 m+2} ; q^{2}\right)_{m} \cdot f_{l}= \\
= & \sum_{r=0}^{\infty} q^{2(m+r)(\alpha+n+1)} \cdot\left(q^{2 r+2} ; q^{2}\right)_{m} \cdot f_{m+r},
\end{aligned}
$$


and

$$
\begin{aligned}
B_{q, \alpha}\left(f_{k}\right)= & \sum_{m=0}^{k} \sum_{r=0}^{\infty} q^{2 \alpha(k-m+r)} \cdot q^{2 k-2 m+2 r n+2 r} . \\
& \cdot \frac{\left(q^{2 \alpha+2} ; q^{2}\right)_{n+m}\left(q^{2 k-2 m+2} ; q^{2}\right)_{n+m-1}\left(q^{2 \alpha+2 n+2} ; q^{2}\right)_{m}\left(q^{2 r+2} ; q^{2}\right)_{m}}{\left(q^{2} ; q^{2}\right)_{n+m-1}\left(q^{2} ; q^{2}\right)_{m}} \cdot f_{m+r} .
\end{aligned}
$$

The substitution $t=q^{2 \alpha}$ gives

$$
\begin{aligned}
B_{q, t}\left(f_{k}\right) & =\sum_{m=0}^{k} \sum_{r=0}^{\infty} t^{k-m+r} \cdot q^{2 k-2 m+2 r n+2 r} . \\
& \cdot \frac{\left(t q^{2} ; q^{2}\right)_{n+m}\left(q^{2 k-2 m+2} ; q^{2}\right)_{n+m-1}\left(t q^{2 n+2} ; q^{2}\right)_{m}\left(q^{2 r+2} ; q^{2}\right)_{m}}{\left(q^{2} ; q^{2}\right)_{n+m-1}\left(q^{2} ; q^{2}\right)_{m}} \cdot f_{m+r} .
\end{aligned}
$$

To finish the proof of (4.14) we have to compute the coefficient at $t$ in the last series. Due to presence of the multiply $t^{k-m+r}$ in the terms, the only terms which might give a non-zero contribution to this coefficient correspond to the values $(m, r)=(k, 0),(m, r)=(k, 1)$, or $(m, r)=(k-1,0)$ which we treat separately

1) $(m, r)=(k, 0)$ : the corresponding term is equal to

$$
\left(t q^{2} ; q^{2}\right)_{n+k}\left(t q^{2 n+2} ; q^{2}\right)_{k} f_{k}=\left(1-t \frac{q^{2 n+2}}{\left(1-q^{2}\right)}\left(1+q^{-2 n}-2 q^{2 k}\right)\right) f_{k}+o(t) .
$$

$2)(m, r)=(k, 1)$ : the corresponding term is equal to

$$
t \cdot q^{2 n+2} \cdot\left(t q^{2} ; q^{2}\right)_{n+k}\left(t q^{2 n+2} ; q^{2}\right)_{k} \frac{1-q^{2 k+2}}{1-q^{2}} f_{k+1}=t \cdot \frac{q^{2 n+2}}{\left(1-q^{2}\right)} \cdot\left(1-q^{2 k+2}\right) f_{k+1}+o(t) .
$$

$3)(m, r)=(k-1,0)$ : the corresponding term is equal to

$t \cdot q^{2} \cdot\left(t q^{2} ; q^{2}\right)_{n+k-1}\left(t q^{2 n+2} ; q^{2}\right)_{k-1} \frac{1-q^{2 n+2 k-2}}{1-q^{2}} f_{k-1}=t \cdot \frac{q^{2 n+2}}{\left(1-q^{2}\right)} \cdot\left(q^{-2 n}-q^{2 k-2}\right) f_{k-1}+o(t)$.

This finishes the proof.

\section{$5 \quad$ Further properties of the $q$-Berezin transform}

In this section we study further the $q$-Berezin transform. Namely, we consider its restriction $B_{q, \alpha}^{(\mathrm{r})}$ onto the space of radial functions. We prove that $B_{q, \alpha}^{(\mathrm{r})}$ is extended to a bounded selfadjoint operator on $\mathcal{L}_{n, q}^{2}$ which commutes with the radial part $\Delta_{n, q}^{(\mathrm{r})}$ of the $q$-LaplaceBeltrami operator. Since the latter has a simple spectrum, $B_{q, \alpha}^{(\mathrm{r})}$ is a function of $\Delta_{n, q}^{(\mathrm{r})}$. We find the function explicitly. We also present an asymptotic expansion for the $q$-Berezin transform at the limit $t=q^{2 \alpha} \rightarrow 0$ mentioned at the end of subsection 4.1 . 


\subsection{Boundedness of the $q$-Berezin transform}

Let $B_{q, \alpha}^{(\mathrm{r})}: \mathcal{L}_{n, q} \rightarrow \mathcal{L}_{n, q}^{\prime}$ be restriction of the $q$-Berezin transform onto the space $\mathcal{L}_{n, q}$ of finite radial functions on the quantum ball.

Proposition $5.1 B_{q, \alpha}^{(\mathrm{r})}$ can be extended to a bounded self-adjoint operator on $\mathcal{L}_{n, q}^{2}$. It is a function of $\Delta_{n, q}^{(\mathrm{r})}$. The operator $\mathcal{F} \cdot B_{q, \alpha}^{(\mathrm{r})} \cdot \mathcal{F}^{-1}$ on $L^{2}\left(\frac{d \rho}{|c(\rho)|^{2}}\right)$ is the multiplication by the (bounded) function

$$
b_{q, \alpha}(\rho)=\frac{\left(q^{2+2 \alpha} ; q^{2}\right)_{\infty} \cdot\left(q^{2 n+2+2 \alpha} ; q^{2}\right)_{\infty}}{\left(q^{n+2+2 \alpha+i \rho} ; q^{2}\right)_{\infty} \cdot\left(q^{n+2+2 \alpha-i \rho} ; q^{2}\right)_{\infty}} .
$$

Proof. We divide the proof into three lemmas.

Lemma $5.2 B_{q, \alpha}^{(\mathrm{r})}\left(\mathcal{L}_{n, q}\right) \subset \mathcal{L}_{n, q}^{2}$ and one has the equality

$$
\Delta_{n, q}^{(\mathrm{r})} \cdot B_{q, \alpha}^{(\mathrm{r})}=B_{q, \alpha}^{(\mathrm{r})} \cdot \Delta_{n, q}^{(\mathrm{r})}
$$

of linear maps from $\mathcal{L}_{n, q}$ to $\mathcal{L}_{n, q}^{2}$.

Sketch of a proof. The inclusion is due to the formula (4.17). Indeed, one has to show that $y^{\alpha+n+1} \cdot\left(y q^{-2 m+2} ; q^{2}\right)_{m} \in \mathcal{L}_{n, q}^{2}$ for any $m$, and this is clear be the definition.

To prove the equality (15.2), we have to show that $\Delta_{n, q}^{(\mathrm{r})} \cdot B_{q, \alpha}^{(\mathrm{r})}\left(f_{k}\right)=B_{q, \alpha}^{(\mathrm{r})} \cdot \Delta_{n, q}^{(\mathrm{r})}\left(f_{k}\right)$ for any $k$. The left-hand side may computed by successive application of (4.17) and (4.5) while the right-hand one may be computed via Proposition 4.3 and (4.17).

Lemma 5.3 Suppose $B$ is a linear operator from $\mathcal{L}_{n, q}$ to $\mathcal{L}_{n, q}^{2}$ which satisfies the properties

$$
\begin{gathered}
\Delta_{n, q}^{(\mathrm{r})} \cdot B=B \cdot \Delta_{n, q}^{(\mathrm{r})} ; \\
B f_{0}=\left(q^{2 \alpha+2} ; q^{2}\right)_{n} \cdot y^{\alpha+n+1} .
\end{gathered}
$$

Then $B=B_{q, \alpha}^{(\mathrm{r})}$.

Proof. The statement is a simple consequence of the equality

$$
\mathcal{L}_{n, q}=\text { linear span of }\left\{\left(\Delta_{n, q}^{(\mathrm{r})}\right)^{m} f_{0}\right\}_{m \in \mathbb{Z}_{\geq 0}}
$$

which in turn may be deduced easily from Proposition 4.3 .

Lemma 5.4 The bounded selfadjoint operator $\mathcal{F}^{-1} \cdot b_{q, \alpha}(\rho) \cdot \mathcal{F}\left(b_{q, \alpha}\right.$ is given by (5.1)) on $\mathcal{L}_{n, q}^{2}$ possesses the properties from the previous lemma. 
Proof.The first property holds trivially. Let us prove the second one.

Let $(\cdot, \cdot)_{L^{2}},(\cdot, \cdot)_{\mathcal{L}^{2}}$ be the inner products in $L^{2}\left(\frac{d \rho}{|c(\rho)|^{2}}\right)$ and $\mathcal{L}_{n, q}^{2}$, respectively. It is sufficient to show that

$$
\left(b_{q, \alpha}(\rho) \cdot \mathcal{F} f_{0}, \mathcal{F} f_{k}\right)_{L^{2}}=\left(q^{2 \alpha+2} ; q^{2}\right)_{n} \cdot\left(y^{\alpha+n+1}, f_{k}\right)_{\mathcal{L}^{2}}
$$

for any $k \in \mathbb{Z}_{\geq 0}$. Recall (4.10) that $\mathcal{F} f_{k}(\rho)=\left(1-q^{2 n}\right) \cdot q^{-k n} \cdot \frac{\left(q^{2 k+2} ; q^{2}\right)_{n-1}}{\left(q^{2 n} ; q^{2}\right)_{k}} \cdot Q_{k}(\rho)$ with $Q_{k}(\rho):=Q_{k}\left(\cos \frac{h \rho}{2} ; q^{n}, q^{n} \mid q^{2}\right)$. We rewrite the condition as follows

$$
\left(b_{q, \alpha}(\rho), Q_{k}(\rho)\right)_{L^{2}}=q^{k(n+2+2 \alpha)} \cdot \frac{\left(q^{2 \alpha+2} ; q^{2}\right)_{n}}{\left(q^{2} ; q^{2}\right)_{n}} .
$$

Remind the following formula for the generating function of the Al-Salam-Chihara polynomials [13, formula (3.8.13)]:

$$
\sum_{j=0}^{\infty} \frac{z^{j}}{\left(q^{2} ; q^{2}\right)_{j}} \cdot Q_{j}(\rho)=\frac{\left(q^{n} z ; q^{2}\right)_{\infty} \cdot\left(q^{n} z ; q^{2}\right)_{\infty}}{\left(q^{i \rho} z ; q^{2}\right)_{\infty} \cdot\left(q^{-i \rho} z ; q^{2}\right)_{\infty}}
$$

Substitution $z:=q^{2 \alpha+2+n}$ gives $\left(q^{2 \alpha+2} ; q^{2}\right)_{n} \sum_{j=0}^{\infty} \frac{q^{j(2 \alpha+n+2)}}{\left(q^{2} ; q^{2}\right)_{j}} \cdot Q_{j}(\rho)=b_{q, \alpha}(\rho)$. Now validity of (5.3) follows from the latter equality and the orthogonality relations (4.13) for the Al-Salam-Chihara polynomials.

Proposition 5.1 follows directly from the last two lemmas.

\subsection{Asymptotic expansion of the $q$-Berezin transform}

Proposition 5.5 For any $f \in \mathcal{D}\left(U_{n}\right)_{q}$

$$
B_{q, t}(f)=\left(q^{2} t ; q^{2}\right)_{n} \cdot \sum_{j=0}^{\infty} t^{j} \cdot q^{2 j} \cdot \frac{\left(q^{2 j+2} ; q^{2}\right)_{n-1}}{\left(q^{2} ; q^{2}\right)_{n-1}} \cdot p_{j}\left(\Delta_{n, q}\right) f
$$

with

$p_{j}\left(\Delta_{n, q}\right)=\sum_{l=0}^{j} \frac{\left(q^{-2 j} ; q^{2}\right)_{l} \cdot q^{2 l}}{\left(q^{2 n} ; q^{2}\right)_{l} \cdot\left(q^{2} ; q^{2}\right)_{l}} \cdot \prod_{m=0}^{l-1}\left(\left(1-q^{2 m}\right)\left(1-q^{2 m+2 n}\right)-q^{2 m+2 n-2}\left(1-q^{2}\right)^{2} \Delta_{n, q}\right)$.

Proof. Recall (see (3.9)) that

$$
B_{q, \alpha}\left(f_{0}\right)=\left(q^{2 \alpha+2} ; q^{2}\right)_{n} \cdot y^{\alpha+n+1}=\left(q^{2 \alpha+2} ; q^{2}\right)_{n} \cdot \sum_{j=0}^{\infty} q^{2 j(\alpha+n+1)} \cdot f_{j}
$$

or, in terms of $t=q^{2 \alpha}$,

$$
B_{q, t}\left(f_{0}\right)=\left(t q^{2} ; q^{2}\right)_{n} \cdot \sum_{j=0}^{\infty} t^{j} q^{2 j(n+1)} \cdot f_{j}
$$


Since any morphism of $U_{q} \mathfrak{s u}_{n, 1}$-modules is determined uniquely by its value on the vector $f_{0}$, it suffices to prove that $p_{j}\left(\Delta_{n, q}\right) f_{0}=q^{2 j n} \cdot \frac{\left(q^{2} ; q^{2}\right)_{n-1}}{\left(q^{2 j+2} ; q^{2}\right)_{n-1}} \cdot f_{j}$ or, equivalently,

$$
p_{j}\left(\Delta_{n, q}^{(\mathrm{r})}\right) f_{0}=q^{2 j n} \cdot \frac{\left(q^{2} ; q^{2}\right)_{n-1}}{\left(q^{2 j+2} ; q^{2}\right)_{n-1}} \cdot f_{j} .
$$

Let us apply the $q$-spherical transform $\mathcal{F}$ to the both hand-sides of the latter equality. We get

$$
p_{j}(\lambda(\rho)) \mathcal{F} f_{0}(\rho)=q^{2 j n} \cdot \frac{\left(q^{2} ; q^{2}\right)_{n-1}}{\left(q^{2 j+2} ; q^{2}\right)_{n-1}} \cdot \mathcal{F} f_{j}(\rho) .
$$

Recall (see (4.10) ) that $\mathcal{F} f_{k}(\rho)=\left(1-q^{2 n}\right) \cdot q^{-2 k n} \cdot\left(q^{2 k+2} ; q^{2}\right)_{n-1} \cdot \phi_{\rho}\left(q^{2 k}\right)$. This formula reduces proving (5.4) to proving the equality

$$
p_{j}(\lambda(\rho))=\phi_{\rho}\left(q^{2 j}\right)
$$

and the latter is just a straightforward computation.

\section{An application: orthogonality relations for contin- uous dual $q$-Hahn polynomials}

The aim of this section is to describe one application of our results to the theory of basic orthogonal polynomials. Namely, we use the $q$-Berezin and the $q$-spherical transform to obtain orthogonality relations for certain two-parameter family of the so-called continuous dual $q$-Hahn polynomials (see [13]). Of course, this result is not new. However we believe that our approach might be interesting.

Throughout this section $\alpha$ is a fixed number.

\subsection{Auxiliary results}

The aim of this subsection is to derive some useful consequences of Proposition 5.1 .

Proposition $6.1 B_{q, \alpha}^{(\mathrm{r})}$ is extended to an invertible operator on $\mathcal{L}_{n, q}^{2}$.

Proof. It suffices to observe that the 'symbol' $b_{q, \alpha}(\rho)$ is invertible:

$$
b_{q, \alpha}(\rho) \geq \frac{\left(q^{2+2 \alpha} ; q^{2}\right)_{\infty} \cdot\left(q^{2 n+2+2 \alpha} ; q^{2}\right)_{\infty}}{\left(-q^{n+2+2 \alpha} ; q^{2}\right)_{\infty} \cdot\left(-q^{n+2+2 \alpha} ; q^{2}\right)_{\infty}} .
$$

To go further, we introduce some auxiliary notations. Denote by $\mathcal{L}_{\text {Op }}$ and $\mathcal{L}_{\text {Op }}^{\prime}$ the subspaces of $U_{q} \mathfrak{s}\left(\mathfrak{u}_{n} \times \mathfrak{u}_{1}\right)$-module morphisms in $\operatorname{End}_{0}\left(\mathbb{C}\left[\mathbb{C}^{n}\right]_{q, \alpha}\right)$ and $\operatorname{End}\left(\mathbb{C}\left[\mathbb{C}^{n}\right]_{q, \alpha}\right)$, respectively. Since the subspaces $\mathbb{C}\left[\mathbb{C}^{n}\right]_{q, \alpha}^{(m)}, m \in \mathbb{Z}_{\geq 0}$, in $\mathbb{C}\left[\mathbb{C}^{n}\right]_{q, \alpha}$ are pairwise non-isomorphic 
irreducible $U_{q} \mathfrak{s}\left(\mathfrak{u}_{n} \times \mathfrak{u}_{1}\right)$-modules (see the proof of lemma 4.6), the vector space $\mathcal{L}_{\text {Op }}$ is generated by the orthogonal projections $P_{m}$ onto the subspaces $\mathbb{C}\left[\mathbb{C}^{n}\right]_{q, \alpha}^{(m)} . \mathcal{L}_{\text {Op }}^{\prime}$ is the space of infinite series of the form

$$
T=\sum_{m} a_{m} P_{m}, \quad a_{m} \in \mathbb{C} .
$$

Elements of $\mathcal{L}_{\mathrm{Op}}$ and $\mathcal{L}_{\text {Op }}^{\prime}$ play the role of 'radial' elements in $\operatorname{End}_{0}\left(\mathbb{C}\left[\mathbb{C}^{n}\right]_{q, \alpha}\right)$ and $\operatorname{End}\left(\mathbb{C}\left[\mathbb{C}^{n}\right]_{q, \alpha}\right)$.

Let $\mathcal{L}_{\text {Op }}^{2}$ be the subspace in $\mathcal{L}_{\text {Op }}^{\prime}$ of ' $q$-Hilbert-Schmidt' operators:

$$
\mathcal{L}_{\mathrm{Op}}^{2}=\left\{T \in \mathcal{L}_{\text {Op }}^{\prime} \mid \operatorname{Tr}_{q}\left(T^{*} \cdot T\right)<\infty\right\} .
$$

Note that the triple $\left(\mathcal{L}_{\text {Op }}, \mathcal{L}_{\text {Op }}^{\prime}, \mathcal{L}_{\text {Op }}^{2}\right)$ is very similar to the triple $\left(\mathcal{L}_{n, q}, \mathcal{L}_{n, q}^{\prime}, \mathcal{L}_{n, q}^{2}\right)$ introduced in subsection 4.3 .

Recall (subsection [3.4) the notation $\sigma$ for the linear map from $\operatorname{End}_{0}\left(\mathbb{C}\left[\mathbb{C}^{n}\right]_{q, \alpha}\right)$ to $\mathcal{D}\left(U_{n}\right)_{q}^{\prime}$ which sends endomorphisms to their covariant symbols. Due to Proposition 3.7 $\sigma\left(\mathcal{L}_{\mathrm{Op}}\right) \subset \mathcal{L}_{n, q}^{\prime}$. Let us denote restriction of $\sigma$ onto $\mathcal{L}_{\mathrm{Op}}$ by $\sigma^{(\mathrm{r})}$.

Proposition 6.2 The image of operator $\sigma^{(\mathrm{r})}$ lies in $\mathcal{L}_{n, q}^{2}$. Moreover, $\sigma^{(\mathrm{r})}$ can be extended to a bounded invertible operator from $\mathcal{L}_{\mathrm{Op}}^{2}$ to $\mathcal{L}_{n, q}^{2}$.

Proof. Recall (subsection [3.4) that the map $\sigma$ is defined via the equality

$$
\int_{U_{n}} \sigma(T) \cdot f d \nu_{q}=\operatorname{Tr}_{q}\left(T \cdot T_{f}\right)
$$

which should be fulfilled for any $f \in \mathcal{D}\left(U_{n}\right)_{q}$. Let $T^{(\mathrm{r})}$ be restriction onto $\mathcal{L}_{n, q}$ of the operator from $\mathcal{D}\left(U_{n}\right)_{q}$ to $\operatorname{End}_{0}\left(\mathbb{C}\left[\mathbb{C}^{n}\right]_{q, \alpha}\right)$ which sends finite functions to the corresponding Toeplitz operators. By Proposition $3.3 T^{(\mathrm{r})}\left(\mathcal{L}_{n, q}\right) \subset \mathcal{L}_{\text {Op }}^{\prime}$. More precisely, by lemma 4.6

$$
T^{(\mathrm{r})}\left(\mathcal{L}_{n, q}\right) \subset \mathcal{L}_{\mathrm{Op}}
$$

In particular, $T^{(\mathrm{r})}$ can be considered as a densely defined operator from $\mathcal{L}_{n, q}^{2}$ into $\mathcal{L}_{\text {Op }}^{2}$. The above definition of $\sigma$ implies the following equivalent definition of $\sigma^{(\mathrm{r})}$ : for an element $T \in \mathcal{L}_{\text {Op }}$ one has $\sigma^{(\mathrm{r})}(T)=t(y)$ iff

$$
\frac{1-q^{2 n}}{1-q^{2}} \int_{0}^{1} t(y) f(y) y^{-n-1}\left(y q^{2} ; q^{2}\right)_{n-1} d_{q^{2}} y=\operatorname{Tr}_{q}\left(T \cdot T^{(\mathrm{r})}(f(y))\right)
$$

for any $f(y) \in \mathcal{L}_{n, q}$. Let us prove that $T^{(\mathrm{r})}$ can be extended up to a bounded operator from $\mathcal{L}_{n, q}^{2}$ to $\mathcal{L}_{\mathrm{Op}}^{2}$. Suppose $f(y) \in \mathcal{L}_{n, q}$. Then, by (6.1) and (6.2)

$$
\operatorname{Tr}_{q}\left(\left(T^{(\mathrm{r})}(f(y))\right)^{*} \cdot T^{(\mathrm{r})}(f(y))\right)=\frac{1-q^{2 n}}{1-q^{2}} \int_{0}^{1} \sigma^{(\mathrm{r})} \cdot T^{(\mathrm{r})}(f(y)) \overline{f(y)} y^{-n-1}\left(y q^{2} ; q^{2}\right)_{n-1} d_{q^{2}} y=
$$




$$
=\frac{1-q^{2 n}}{1-q^{2}} \int_{0}^{1} B_{q, \alpha}^{(\mathrm{r})}(f(y)) \overline{f(y)} y^{-n-1}\left(y q^{2} ; q^{2}\right)_{n-1} d_{q^{2}} y=\left(B_{q, \alpha}^{(\mathrm{r})} f, f\right)_{\mathcal{L}^{2}} \leq\left\|B_{q, \alpha}^{(\mathrm{r})}\right\|(f, f)_{\mathcal{L}^{2}}
$$

To prove boundedness of $\sigma^{(\mathrm{r})}$ it remains to observe that, by $(6.2), \sigma^{(\mathrm{r})}$ coincides on $\mathcal{L}_{\text {Op }}$ with $\left(T^{(\mathrm{r})}\right)^{*}$. Invertibility of $\sigma^{(\mathrm{r})}$ may be deduced using similar arguments and Proposition 6.1 .

\subsection{Orthogonality relations}

First we describe an idea of producing the orthogonality relations. It is based on the following statement.

Proposition 6.3 The operator

$$
U_{\alpha}: \mathcal{L}_{\mathrm{Op}}^{2} \rightarrow L^{2}\left(\frac{d \rho}{|c(\rho)|^{2}}\right), \quad T \mapsto \frac{1}{\sqrt{b_{q, \alpha}(\rho)}} \cdot \mathcal{F} \sigma^{(\mathrm{r})}(T)
$$

is unitary.

Remark. In the classical case the operator $U_{\alpha}$ (the product of the spherical transform with the unitary part of the covariant symbol map) was studied in [14.

Proof. By Proposition 5.1

$$
\frac{1}{b_{q, \alpha}(\rho)} \cdot \mathcal{F}=\mathcal{F}\left(B_{q, \alpha}^{(\mathrm{r})}\right)^{-1}
$$

Remind the notation $(\cdot, \cdot)_{L^{2}},(\cdot, \cdot)_{\mathcal{L}^{2}}$ for the inner products in $L^{2}\left(\frac{d \rho}{|c(\rho)|^{2}}\right)$ and $\mathcal{L}_{n, q}$, respectively. Then

$$
\begin{gathered}
\left(\frac{1}{\sqrt{b_{q, \alpha}(\rho)}} \cdot \mathcal{F} \sigma^{(\mathrm{r})}(T), \frac{1}{\sqrt{b_{q, \alpha}(\rho)}} \cdot \mathcal{F} \sigma^{(\mathrm{r})}(T)\right)_{L^{2}}= \\
=\left(\frac{1}{b_{q, \alpha}(\rho)} \cdot \mathcal{F} \sigma^{(\mathrm{r})}(T), \mathcal{F} \sigma^{(\mathrm{r})}(T)\right)_{L^{2}}=\left(\mathcal{F}\left(B_{q, \alpha}^{(\mathrm{r})}\right)^{-1} \sigma^{(\mathrm{r})}(T), \mathcal{F} \sigma^{(\mathrm{r})}(T)\right)_{L^{2}}= \\
=\left(\left(B_{q, \alpha}^{(\mathrm{r})}\right)^{-1} \sigma^{(\mathrm{r})}(T), \sigma^{(\mathrm{r})}(T)\right)_{\mathcal{L}^{2}} .
\end{gathered}
$$

The result then follows since $\left(B_{q, \alpha}^{(\mathrm{r})}\right)^{-1}=\left(\sigma^{(\mathrm{r}) *}\right)^{-1} \cdot\left(\sigma^{(\mathrm{r})}\right)^{-1}$.

Recall that the projections $P_{m}, m \in \mathbb{Z}_{\geq 0}$ constitute an orthogonal basis in the Hilbert space $\mathcal{L}_{O p}^{2}$. Proposition 6.3 implies that $\mathcal{F} \sigma^{(\mathrm{r})}\left(P_{m}\right), m \in \mathbb{Z}_{\geq 0}$ constitute an orthogonal basis in $L^{2}\left(\frac{d \rho}{b_{q, \alpha}(\rho)|c(\rho)|^{2}}\right)$. Our intention now is to show that $\mathcal{F} \sigma^{(\mathrm{r})}\left(P_{m}\right)$ are very close to certain continuous dual $q$-Hahn polynomials, and the above observations will give us the orthogonality relations for these polynomials. 
Proposition 6.4 Let $P_{m}(\rho)=\mathcal{F} \sigma^{(\mathrm{r})}\left(P_{m}\right)(\rho)$. Then

$$
\begin{gathered}
P_{m}(\rho)=\frac{\left(q^{2} ; q^{2}\right)_{n} \cdot\left(q^{2 n+2+2 \alpha} ; q^{2}\right)_{\infty}^{2}}{\left(q^{n+2+2 \alpha+i \rho} ; q^{2}\right)_{\infty} \cdot\left(q^{n+2+2 \alpha-i \rho} ; q^{2}\right)_{\infty}} \cdot q^{-2 m(n+\alpha)} \cdot \frac{\left(q^{2 n+2+2 \alpha} ; q^{2}\right)_{m}}{\left(q^{2} ; q^{2}\right)_{m}} \\
\cdot{ }_{3} \phi_{2}\left(\begin{array}{c}
q^{-2 m}, \quad q^{n+2+2 \alpha+i \rho}, \quad q^{n+2+2 \alpha-i \rho} \\
q^{2 n+2+2 \alpha}, \quad q^{2 n+2+2 \alpha}
\end{array} q^{2}, \quad q^{2}\right) .
\end{gathered}
$$

Proof. Remind (lemma 4.7) that we have already computed $\sigma^{(\mathrm{r})}\left(P_{m}\right)$

$$
\sigma^{(\mathrm{r})}\left(P_{m}\right)=q^{-2 m(\alpha+n+1)} \cdot \frac{\left(q^{2 \alpha+2 n+2} ; q^{2}\right)_{m}}{\left(q^{2} ; q^{2}\right)_{m}} \cdot y^{\alpha+n+1} \cdot\left(y q^{-2 m+2} ; q^{2}\right)_{m} .
$$

By the $q$-binomial formula [20] the equality can be rewritten as follows

$$
\begin{aligned}
\sigma^{(\mathrm{r})}\left(P_{m}\right)= & q^{-2 m(\alpha+n+1)} \cdot \frac{\left(q^{2 \alpha+2 n+2} ; q^{2}\right)_{m}}{\left(q^{2} ; q^{2}\right)_{m}} \cdot \sum_{l=0}^{m} \frac{\left(q^{2} ; q^{2}\right)_{m}}{\left(q^{2} ; q^{2}\right)_{l}\left(q^{2} ; q^{2}\right)_{m-l}} \cdot(-1)^{m} \cdot q^{l(l-2 m+1)} \cdot y^{n+1+l+\alpha}= \\
& =q^{-2 m(\alpha+n+1)} \cdot \sum_{l=0}^{m} \frac{\left(q^{2 \alpha+2 n+2} ; q^{2}\right)_{m}}{\left(q^{2} ; q^{2}\right)_{l}\left(q^{2} ; q^{2}\right)_{m-l}} \cdot(-1)^{m} \cdot q^{l(l-2 m+1)} \cdot y^{n+1+l+\alpha} \cdot
\end{aligned}
$$

Hence it remains to compute $\mathcal{F} y^{n+1+l+\alpha}$ for any $l \geq 0$. Let us apply the $q$-spherical transform to the both hand-sides of the formula $B_{q, \alpha}\left(f_{0}\right)=\left(q^{2 \alpha+2} ; q^{2}\right)_{n} \cdot y^{\alpha+n+1}$. Due to Proposition 5.1

$$
\frac{\left(q^{2+2 \alpha} ; q^{2}\right)_{\infty} \cdot\left(q^{2 n+2+2 \alpha} ; q^{2}\right)_{\infty}}{\left(q^{n+2+2 \alpha+i \rho} ; q^{2}\right)_{\infty} \cdot\left(q^{n+2+2 \alpha-i \rho} ; q^{2}\right)_{\infty}} \cdot \mathcal{F} f_{0}=\left(q^{2+2 \alpha} ; q^{2}\right)_{n} \cdot \mathcal{F} y^{n+1+\alpha},
$$

or, equivalently,

$$
\mathcal{F} y^{n+1+\alpha}=\frac{\left(q^{2} ; q^{2}\right)_{n} \cdot\left(q^{2 n+2+2 \alpha} ; q^{2}\right)_{\infty}^{2}}{\left(q^{n+2+2 \alpha+i \rho} ; q^{2}\right)_{\infty} \cdot\left(q^{n+2+2 \alpha-i \rho} ; q^{2}\right)_{\infty}} .
$$

Let us make the substitution $N:=n+1+\alpha(N>n+1)$ :

$$
\mathcal{F} y^{N}=\frac{\left(q^{2} ; q^{2}\right)_{n} \cdot\left(q^{2 N} ; q^{2}\right)_{\infty}^{2}}{\left(q^{2 N-n+i \rho} ; q^{2}\right)_{\infty} \cdot\left(q^{2 N-n-i \rho} ; q^{2}\right)_{\infty}}
$$

Thus,

$$
\mathcal{F} y^{n+1+l+\alpha}=\frac{\left(q^{2} ; q^{2}\right)_{n} \cdot\left(q^{2 n+2+2 l+2 \alpha} ; q^{2}\right)_{\infty}^{2}}{\left(q^{n+2+2 l+2 \alpha+i \rho} ; q^{2}\right)_{\infty} \cdot\left(q^{n+2+2 l+2 \alpha-i \rho} ; q^{2}\right)_{\infty}} .
$$

The latter equality, together with (6.3) and simple computations, gives our formula.

Recall the notation $p_{k}(x ; a, b, c \mid q)$ for the continuous dual $q$-Hahn polynomials [13, Section 3.3]. The above proposition implies

$$
\begin{aligned}
& \mathcal{F} \sigma^{(\mathrm{r})}\left(P_{m}\right)= \\
= & \frac{q^{-m n} \cdot\left(q^{2} ; q^{2}\right)_{n} \cdot\left(q^{2 n+2+2 \alpha} ; q^{2}\right)_{\infty} \cdot\left(q^{2 n+2 m+2+2 \alpha} ; q^{2}\right)_{\infty}}{\left(q^{n+2+2 \alpha+i \rho} ; q^{2}\right)_{\infty} \cdot\left(q^{n+2+2 \alpha-i \rho} ; q^{2}\right)_{\infty} \cdot\left(q^{2} ; q^{2}\right)_{m}} \cdot p_{m}\left(\cos \frac{h \rho}{2} ; q^{n+2+2 \alpha}, q^{n}, q^{n} \mid q^{2}\right) .
\end{aligned}
$$


Let $p_{m}(\rho):=p_{m}\left(\cos \frac{h \rho}{2} ; q^{n+2+2 \alpha}, q^{n}, q^{n} \mid q^{2}\right)$. Applying Proposition 6.3 and above observations, we get

$$
\begin{aligned}
& \frac{1}{4 \pi} \int_{0}^{\frac{2 \pi}{h}} p_{m}(\rho) p_{l}(\rho) \frac{d \rho}{\left(q^{n+2+2 \alpha+i \rho} ; q^{2}\right)_{\infty}^{2} \cdot\left(q^{n+2+2 \alpha-i \rho} ; q^{2}\right)_{\infty}^{2} \cdot b_{q, \alpha}(\rho)|c(\rho)|^{2}}= \\
& \quad=\frac{q^{2 m n} \cdot\left(1-q^{2 n}\right) \cdot\left(q^{2} ; q^{2}\right)_{m}^{2}}{h \cdot\left(q^{2} ; q^{2}\right)_{n}^{2} \cdot\left(q^{2 n+2+2 \alpha} ; q^{2}\right)_{\infty}^{2} \cdot\left(q^{2 n+2 m+2+2 \alpha} ; q^{2}\right)_{\infty}^{2}} \cdot \operatorname{Tr}_{q}\left(P_{m} \cdot P_{l}\right),
\end{aligned}
$$

or, using the explicit formula for $b_{q, \alpha}$,

$$
\begin{gathered}
\frac{1}{4 \pi} \int_{0}^{\frac{2 \pi}{h}} p_{m}(\rho) p_{l}(\rho) \frac{d \rho}{\left(q^{n+2+2 \alpha+i \rho} ; q^{2}\right)_{\infty}\left(q^{n+2+2 \alpha-i \rho} ; q^{2}\right)_{\infty}|c(\rho)|^{2}}= \\
=\delta_{m l} \frac{\left(q^{2} ; q^{2}\right)_{m}\left(q^{2} ; q^{2}\right)_{m+n-1}}{h\left(q^{2} ; q^{2}\right)_{n-1}\left(q^{2 n+2 m+2+2 \alpha} ; q^{2}\right)_{\infty}^{2}} .
\end{gathered}
$$

Recall (Proposition 4.5) that

$$
\frac{1}{|c(\rho)|^{2}}=\left|\frac{\Gamma_{q^{2}}^{2}\left(\frac{n}{2}+\frac{i \rho}{2}\right)}{\Gamma_{q^{2}}(n) \Gamma_{q^{2}}(i \rho)}\right|^{2}=\left(q^{2 n} ; q^{2}\right)_{\infty}^{2} \cdot \frac{\left(q^{2 i \rho} ; q^{2}\right)_{\infty}\left(q^{-2 i \rho} ; q^{2}\right)_{\infty}}{\left(q^{n+i \rho} ; q^{2}\right)_{\infty}^{2}\left(q^{n-i \rho} ; q^{2}\right)_{\infty}^{2}} .
$$

Finally we have

$$
\begin{gathered}
\frac{1}{4 \pi} \int_{0}^{\frac{2 \pi}{h}} p_{m}(\rho) p_{l}(\rho) \frac{\left(q^{2 i \rho} ; q^{2}\right)_{\infty}\left(q^{-2 i \rho} ; q^{2}\right)_{\infty}}{\left(q^{n+2+2 \alpha+i \rho} ; q^{2}\right)_{\infty}\left(q^{n+2+2 \alpha-i \rho} ; q^{2}\right)_{\infty}\left(q^{n+i \rho} ; q^{2}\right)_{\infty}^{2}\left(q^{n-i \rho} ; q^{2}\right)_{\infty}^{2}} d \rho= \\
=\delta_{m l} \frac{1}{h\left(q^{2 n+2 m+2+2 \alpha} ; q^{2}\right)_{\infty}^{2}\left(q^{2 m+2} ; q^{2}\right)_{\infty}\left(q^{2 n+2 m} ; q^{2}\right)_{\infty}} .
\end{gathered}
$$

The latter equality is a particular case of the orthogonality relations for the entire family of continuous dual $q$-Hahn polynomials given in [13, (3.3.2)].

\section{Conclusion}

As it was mentioned in the Introduction, this research is, above all, a part of the general program of studying $q$-Cartan domains, which we believe is a promising subject in quantum group theory. To our knowledge the results above on finding a mathematical setting for the Toeplitz and covariant calculi, expressing Berezin transform as a function of the $q$-Laplacian operator, and on computing the covariant symbol of the projections are all new. Those results, in the classical case, are naturally related to finding an expansion of the associated $*$-product $f *_{h} g$ of covariant symbols as a power series in the Planck 
constant $h$ (see e. g. 25]). Thus, the most natural continuation of the present paper would be understanding the expansion of the $*$-product in this case since certain computation might be easier than in the classical case, and generalization of our results to the case of other, more complicated $q$-Cartan domains, first of all, $q$-matrix balls [9, 10]. We mention the paper [10] where $q$-weighted Bergman spaces on $q$-matrix balls are constructed and studied. Using these results, it is not so difficult to generalize our definition of the $q$-Berezin transform. However, to obtain more deep results (for instance, an explicit asymptotic formula for the $q$-Berezin transform) one needs to develop to some extent harmonic analysis on $q$-matrix balls. This seems to be a difficult problem itself. By now, harmonic analysis is developed quite well for compact quantum homogeneous spaces only. Nevertheless, we are fully confident that all the results we present in the paper admit generalization to other $q$-Cartan domains.

\section{References}

[1] S. Sinel'shchikov and L. Vaksman, On q-analogues of bounded symmetric domains and Dolbeault complexes, Math. Phys., Anal., and Geom. 1, 1998, 75 - 100; E-print: q-alg/9703005.

[2] L.-K. Hua. Harmonic analysis of functions of several complex variables in the classical domains, Transl. Math. Mono, Vol. 6, Amer. Math. Soc., Providence, 1963.

[3] F. A. Berezin. General concept of quantization, Comm. Math. Phys. 40, 1975, 153 174.

[4] F. A. Berezin. On relations between covariant and contravariant symbols of operators for complex classical domains, Sov. Math. Dokl. 19, 1978, 786 - 789.

[5] A. Unterberger and H. Upmeier, The Berezin transform and invariant differential operators, Comm. Math. Phys. 164, 1994, 563 - 597.

[6] S. Klimek, A. Lesniewski. A two-parameter quantum deformation of the unit disc, J. Funct. Anal. 115, 1993, $1-23$.

[7] D. Shklyarov, S. Sinel'shchikov, and L. Vaksman. On function theory in quantum disc: a q-analogue of the Berezin transform, E-print: math.QA/9809018

[8] D. Shklyarov, S. Sinel'shchikov, and L. Vaksman. A q-analogue of the Berezin quantization method, Letters in Mathematical Physics 49, 1999, No. 3, 253 - 261.

[9] D. Shklyarov, S. Sinel'shchikov, L. Vaksman. Quantum matrix ball: differential and integral calculi, E-print: QA/9905035. 
[10] D. Shklyarov, S. Sinel'shchikov, L. Vaksman. Quantum matrix ball: The Bergman kernel, E-print: QA/9909036.

[11] H. T. Koelink, J. V. Stokman. Fourier transforms on the quantum $S U(1,1)$ group, E-print: QA/9911163.

[12] S. Helgason. Groups and Geometric Analysis, Academic Press Inc., N.Y. - London, 1984.

[13] R. Koekoek, R. F. Swarttouw. The Askey-scheme of hypergeometric orthogonal polynomials and its q-analogue, Preprint Delft Univ. of Technology, Report no. 98-17, 1998.

[14] J. Peetre and G. Zhang, A weighted Plancherel formula III. The case of a hyperbolic matrix domain, Collect. Math. 43, 1992, 273 - 301.

[15] Yu. A. Neretin. Index hypergeometric transform and immitation of analysis of Berezin kernels on hyperbolic spaces, E-print: CA/0104035.

[16] W. Pusz, S. Woronowicz, Twisted second quantization, Reports Math. Phys. 27, 1989, $231-257$.

[17] V. Chari, A. Pressley. A Guide to Quantum Groups, Cambridge Univ. Press, 1995.

[18] D. Proskurin, Yu. Samoilenko. Stability of the $C^{*}$-algebra associated with the twisted CCR, E-print: OA/0112284.

[19] D. Shklyarov, S. Sinel'shchikov, and L. Vaksman. On function theory in quantum disc: integral representations, E-print: QA/9808015.

[20] G. Gasper, M. Rahman. Basic hypergeometric series, Cambridge Univ. Press, 1995.

[21] N. Wallach. The analytic continuation of the discrete series, I, II, Trans. Amer. Math. Soc. 251, 1979, 1 - 17; $19-37$.

[22] J. Arazy. A Survey of Invariant Hilbert Spaces of Analytic Functions on Bounded Symmetric Domains, Contemp. Math. 185, 1995, 7 - 64.

[23] G. van Dijk, S. C. Hille. Canonical representations related to hyperbolic spaces, J. Funct. Anal. 147, 1997, $109-139$.

[24] J. Peetre. Berezin transform and Ha-plitz opertors, J. Oper. Theory 24, 1990, 165 168.

[25] J. Oesterlé. Quantification formelle des variétés de Poisson (d'après Maxim Kontsevich), Astérisque, 252, 1998, Exp. No. 843, 4, 211-229. 